\title{
Arabidopsis sirtuins and poly ADP ribose polymerases regulate gene expression in the day but do not affect circadian rhythms
}

\author{
Jun Hyeok Kim ${ }^{1}$, Laura Bell ${ }^{1}$, Xiao Wang ${ }^{1}$, Rinukshi Wimalasekera ${ }^{1}$, Hugo Bastos ${ }^{1}$, \\ Krystyna Kelly ${ }^{1}$, Matthew Hannah ${ }^{2}$, and Alex Webb ${ }^{1}$ \\ ${ }^{1}$ University of Cambridge \\ ${ }^{2}$ Bayer CropScience NV
}

November 11, 2020

\begin{abstract}
Nicotinamide-adenine dinucleotide (NAD) is involved in redox homeostasis and acts as a substrate for NADases, including poly (ADP-ribose) polymerases (PARPs) that add poly (ADP-ribose) polymers to proteins and DNA, and sirtuins that deacetylate proteins. Nicotinamide, a biproduct of NADases increases circadian period in both plants and animals. In mammals, the effect of nicotinamide on circadian period might be mediated by the PARPs and sirtuins because thy directly bind to core circadian oscillator genes. We have investigated whether PARPs and sirtuins contribute to the regulation of the circadian oscillator in Arabidopsis. We found no evidence that PARPs and sirtuins regulate the circadian oscillator of Arabidopsis or are involved in the response to nicotinamide. RNA-seq analysis indicated that PARPs regulate the expression of only a few genes, including FLOWERING LOCUS C. However, we found profound effects of reduced sirtuin 1 expression on gene expression during the day but not at night, and an embryo lethal phenotype in knockouts. Our results demonstrate that PARPS and sirtuins are not associated with NAD regulation of the circadian oscillator and that sirtuin 1 is associated with daytime regulation of gene expression.
\end{abstract}

Short title: Sirtuin 1 regulates day time gene expression

Corresponding author: Alex A.R. Webb, aarw2@cam.ac.uk

Article Title:Arabidopsis sirtuins and poly ADP ribose polymerases regulate gene expression in the day but do not affect circadian rhythms

Jun Hyeok Kim, Laura J. Bell, Xiao Wang, Rinuckshi Moeller, Hugo P. Bastos, Krystyna A. Kelly, Matthew A. Hannah ${ }^{1}$ and Alex A.R. Webb

Department of Plant Sciences, University of Cambridge, Downing Street, Cambridge, CB3 0LJ, UK.

${ }^{1}$ BASF, BBCC - Innovation Center Gent, Technologiepark-Zwijnaarde 101, 9052 Gent, Belgium.

List of author contributions

J.H.K., L.J.B., X.W. and R.M. performed the experimentation. H.B. and K.K. performed the bioinformatics. M.A.H. and A.A.R.W. conceived the research and obtained funding. J.H.K., L.J.B. and A.A.R.W. wrote the paper.

\section{Funding}

L.J.B. was supported by a BBSRC Industrial CASE studentship awarded to M.A.H. and A.A.R.W. J.H.K., X.W. and H.B. were supported by BBSRC grant BB/L02182X/1 awarded to K.K. and A.A.R.W. 


\begin{abstract}
Nicotinamide-adenine dinucleotide (NAD) is involved in redox homeostasis and acts as a substrate for NADases, including poly (ADP-ribose) polymerases (PARPs) that add poly (ADP-ribose) polymers to proteins and DNA, and sirtuins that deacetylate proteins. Nicotinamide, a biproduct of NADases increases circadian period in both plants and animals. In mammals, the effect of nicotinamide on circadian period might be mediated by the PARPs and sirtuins because thy directly bind to core circadian oscillator genes . We have investigated whether PARPs and sirtuins contribute to the regulation of the circadian oscillator in Arabidopsis. We found no evidence that PARPs and sirtuins regulate the circadian oscillator of Arabidopsis or are involved in the response to nicotinamide. RNA-seq analysis indicated that PARPs regulate the expression of only a few genes, including FLOWERING LOCUS C . However, we found profound effects of reduced sirtuin 1 expression on gene expression during the day but not at night, and an embryo lethal phenotype in knockouts. Our results demonstrate that PARPS and sirtuins are not associated with NAD regulation of the circadian oscillator and that sirtuin 1 is associated with daytime regulation of gene expression.
\end{abstract}

\title{
Keywords
}

Arabidopsis thaliana , Nicotinamide-adenine dinucleotide, poly (ADP-ribose) polymerases, Sirtuins, poly(ADP-ribose) glycohydrolase, Circadian clock

\section{Introduction}

Plant life is heavily influenced by the cycles of light, dark and temperature caused by the rotation of the planet on its axis, resulting in rhythmic outputs modulated by the circadian clock (Webb et al2019). Metabolism is an important output of the circadian system in plants, with the circadian oscillator regulating the expression and activity of the components of many metabolic pathways, including photosynthesis and starch metabolism (Dodd et al ., 2005; Grafet al ., 2010; Lu et al ., 2005), nutrient assimilation (Gutierez et al ., 2008), redox homeostasis (Lai et al ., 2012) and secondary metabolism (Kerwin et al ., 2011). However, metabolism has recently become recognized also to act as a crucial input regulating the circadian oscillators of both plants and animals. For example, in Arabidopsis, the period of the circadian oscillator is regulated by sugars (Haydon et al ., 2013; Frank et al ., 2018) and nicotinamide (Dodd et al ., 2007; Mombaerts et al ., 2019). Sugars fluctuate as a consequence of carbon homeostasis and photosynthesis (Webb et al ., 2019) whereas nicotinamide is a breakdown product of NAD acting as a substrate for post-translational modifications including poly(ADP-ribosyl)ation and protein deacetylation, and for the production of the $\mathrm{Ca}^{2+}$ agonist cyclic ADP ribose (cADPR) (Hunt et al ., 2004). Nicotinamide lengthens circadian period in all organisms tested with proposed modes of action being through inhibition of poly (ADP-ribose) polymerases (PARPs), sirtuins (SRTs), ADPR cyclases, the reduction of H3K4me3 accumulation, TARGET OF RAPAMYCIN (TOR) and the action of BIG, a protein of unknown function (Dodd et al ., 2007; Asher et al ., 2008; Nakahata et al ., 2008; Asher et al ., 2010; Malapeira et al ., 2012; Hearn et al ., 2018; Mombaertset al ., 2019; Zhang et al . 2019).

In mammals, PARP1 participates in the phase entrainment of peripheral clocks (Asher et al ., 2010) and mice lacking PARP1 have a phase shift in the timing of complex formation between the circadian oscillator components CLOCK/BMAL1 and PER, and the entrainment of the peripheral circadian clock in the liver to inverted feeding cycles was significantly delayed (Kumar \& Takahashi., 2010). PARPs also play roles in DNA repair, maintenance of genomic stability, transcription, chromatin structure, cell cycle and telomere length, energy metabolism and cell death (Krishnakumar \& Kraus, 2010; Schreiber et al ., 2006). In plants, PARPs play a crucial role in the innate immune responses because parp1parp2 double mutant Arabidopsis have compromised immune gene activation and enhanced susceptibility to pathogen infections (Feng et al ., 2015). Decreased levels of PARP activity by chemical inhibition or genetic downregulation have been correlated with increased tolerance to abiotic stresses including oxidative, drought and heat stress (De Block et al., 2005; Vanderauwera et al., 2007) but parp loss-of-function mutants are not affected in abiotic stress responses (Rissel et al., 2017). Therefore, the role of PARPs in plant abiotic stress responses is still an open question and their effect on circadian function in plants has not been reported. 
PARP enzymatic activity is counteracted by the enzyme poly(ADP-ribose) glycohydrolase (PARG), which hydrolyses PAR polymers and releases ADP-ribose subunits. Consistent with a role for PARPs in immune responses in plants, both PARG1 and PARG2 are required for stress responses to Botrytis cinerea in tomato and an increase in ADP-ribose polymer levels was observed in response to avirulent Pst DC3000 (AdamsPhillips et al., 2010). Unlike the PARPs, PARG activity has been shown to regulate the circadian oscillator of Arabidopsis because the TEJ1 mutation in PARG1 has long free-running circadian period (Panda et al ., 2002).

Sirtuins, like the PARPs are NADases whose enzymatic activity is also inhibited by nicotinamide. Sirtuins are $\mathrm{NAD}^{+}$-dependent protein deacetylases homologous to the yeast Sir2 protein, which is responsible for heterochromatin formation in yeast (Imai et al ., 2000; Landry et al ., 2000; Smith et al ., 2000). In mammals, SIRTUIN1 (SIRT1) is closely associated with core circadian oscillator components. SIRT1 activity is rhythmic, and cultured mouse cells null for SIRT1 or transfected with SIRT1 siRNA had reduced amplitude of the BMAL1:LUC circadian oscillator reporter, suggesting SIRT1 is required to maintain the magnitude of oscillator gene expression (Asher et al ., 2008).

We have found evidence that nicotinamide affects the Arabidopsis circadian oscillator through its action on ADPRcyclase and the inhibition of Ca signalling (Dodd et al ., 2007; Awal et al2016; Marti et al 2018; Hearn et al ., 2018; Mombeartset al 2019). Here we have tested the counter hypothesis that members of the Arabidopsis PARP and $S R T$ gene families participate in circadian regulation, and that this might explain the mode of action of nicotinamide in the circadian system. We performed this study because of the important role of the PARPs and SRTs in mammalian systems and the potential that this might be a conserved function between the circadian systems across Kingdoms. Using a combination of genetic and transcriptome analysis we find that the PARPs and SRTs are unlikely to be involved in circadian regulation in Arabidopsis and do not appear to explain the response of the Arabidopsis circadian oscillator to nicotinamide. These data define a major regulatory difference between the plant and mammalian circadian oscillators. We identify a function for PARPs in photoperiodic responses through the regulation of the flowering time regulator FLOWERING LOCUS C (FLC). We demonstrate that SRT1 function might have been obscured in previous analysis of Arabidopsis because srt 1 knockouts are embryo lethal, which we conclude might be related to a profound effect of srt1 knockdown on gene expression during the day.

\section{Material and methods}

\section{Plant material and growth conditions}

Surface sterilised Arabidopsis thaliana seeds were sown directly onto half strength Murashige and Skoog (MS) (Duchefa Biochemie, Netherlands) $0.8 \%$ bactoagar (BD, USA). Stratification at $4^{\circ} \mathrm{C}$ in the dark was for two days, before transfer to Sanyo (UK) growth cabinets $\left(19-22^{\circ} \mathrm{C}, 100 \mu \mathrm{mol} \mathrm{m}{ }^{-2} \mathrm{~s}^{-2} ; 12\right.$ hours light/12 hours dark.

T-DNA lines used in this investigation are described in Supplemental Table 1. Genotyping was carried out according to the instructions athttp://signal.salk.edu/tdnaprimers.2.htmlusing the primers listed in Supplemental Table 2. Left border PCR products were sequenced to determine the precise locations of the T-DNA inserts.

For srt1-4 heterozygous (hete) and srt1-4 hete $x$ 2-1 mutants, we performed genotyping by PCR to identify heterozygous plants for each experiment. SRT1 artificial miRNAs were designed using the MicroRNA Designer tool of the WMD3 Web site (Ossowski et al., 2008) and were introduced by transformation as described below.

\section{Leaf movement imaging and analysis}

A. thaliana seeds were sown in-vitro individually, with an approximately $1 \mathrm{~cm}$ gap in between each seed. To promote hypocotyl elongation plants were grown under the same condition as described above in individual $5 \mathrm{~cm}$ tall cardboard boxes for 10 - 12 days. Individual seedlings were cut out in $1 \mathrm{~cm}^{2}$ blocks of agar with a scalpel and transferred to vertical standing 25 -well $10 \mathrm{~cm}^{2}$ culture dishes (Sterilin, UK) in constant 
white light $\left(100 \mu \mathrm{mol} \mathrm{m} \mathrm{m}^{-2} \mathrm{~s}^{-2}\right)$. Circadian rhythms of leaf movement were recorded using time-lapse image capture from an array of video cameras controlled by Metamorph ${ }^{\mathrm{TM}}$ software (Molecular Devices LLS, USA). Images were captured every 20 minutes for seven days. Leaf position data were extracted from images using Metamorph $^{\mathrm{TM}}$ software. Rhythmic traces were analysed using Biological Rhythms Analysis Software System (BRASS) software package which obtains period estimates and relative amplitude error (RAE) values using a Fast-Fourier Transform non-linear least squares (FFT-NLS) method. The first 24 hours of data were excluded from analysis, and movements were considered rhythmic if they had a period between 15 and 35 hours.

\section{Photoperiodic flowering time screening}

Sixteen plants per line were sown into soil trays with 24 cells with the positions being allocated randomly. The seeds were stratified for three days at $4{ }^{\circ} \mathrm{C}$ before transfer to $20 \mathrm{deg} \mathrm{C}$ and $100 \mu \mathrm{mol} \mathrm{m} \mathrm{m}^{-2} \mathrm{~s}^{-1}$ in either long day (LD) conditions (16 h L:8 h D) or short day (SD) conditions ( $8 \mathrm{~h} \mathrm{~L}: 16 \mathrm{~h} \mathrm{D})$. Seed trays were moved regularly to prevent any positional effects on plant growth. The time to bolt and the number of rosette leaves at the time of bolting were recorded. Bolting time was measured as the number of days from sowing when the inflorescence stem was approximately $5 \mathrm{~mm}$ tall.

\section{Transformation of Arabidopsis with pCCA1::LUC ${ }^{+}$}

Agrobacterium tumefaciens (Gv3101) transformed with appropriate constructs (Ti plasmid: pPCVH containing $L U C+$; helper plasmid: pM90RK; CCA1:luc binary vector), a gift from A. Millar (University of Edinburgh) were inoculated into $5 \mathrm{ml}$ Luria Bertani cultures containing gentamycin $\left(25 \mu \mathrm{g} \mathrm{ml}^{-1}\right)$ and ampicillin $\left(50 \mu \mathrm{g} \mathrm{ml}{ }^{-1}\right)$ two days before transformation. After cultures were left overnight at $30{ }^{\circ} \mathrm{C}, 2.5 \mathrm{ml}$ was sub-cultured into a $50 \mathrm{ml}$ culture, and then grown at $30{ }^{\circ} \mathrm{C}$ overnight. The culture was generously painted onto whole plants (leaves, secondary stems and inflorescences) with an autoclaved paintbrush. Plants were covered in a cling film tent for two days to increase humidity.

\section{Measurement of circadian rhythms using luminescent circadian clock reporter gene fusions}

Seeds were sown on to $0.5 \mathrm{MS} 0.8 \%$ agar in PVC tubing rings ( $0.7 \mathrm{~mm}$ diameter) in clusters of $2-5$ seeds for imaging of luciferase. Plants were grown under same condition as described above for 10-12 days before imaging. Twenty-four and 48 hours before imaging seedlings were dosed with $50 \mu \mathrm{l}$ of $2 \mathrm{mM}$ luciferin, the substrate of luciferase. Plants were imaged using Photek ICCD225 photon counting cameras using IFS32 software. Automated images were captured every hour for 800 s for luciferase imaging, or for 1500 s every 2 hours for imaging of aequorin. In order to analyse images, captured regions were drawn on a pseudo-coloured image around the sites of clusters using IFS32 software, with the bright field image used as a reference. Total photon counts (luminescence) per image per unit time were extracted. Period estimates were obtained using BRASS software.

\section{Delayed chlorophyll fluorescence}

Seeds were sown in clusters of approximately 20 seeds, one cluster per well in a $10 \mathrm{~cm}$ square 25 well plate. The dividing walls of the plate were covered in black electrical tape, to reduce cross contamination of signal between wells. After 10 - 12 days of entrainment, plates were transferred into a NightSHADE LB 985 photon counting camera (Berthold Technologies), controlled by IndiGO ${ }^{\mathrm{TM}}$ software. Plants were grown in the camera box for one light/dark cycle before switching to constant light conditions for imaging. Illumination in the camera was maintained by light emitting diodes (LEDs) set to $24 \%$ of maximum emission for red $(660 \mathrm{~nm})$ and blue $(470 \mathrm{~nm})$ wavelengths to provide light intensity $50-70 \mu \mathrm{mol} \mathrm{m} \mathrm{m}^{-2} \mathrm{~s}^{-2}$ at plant height. Delayed fluorescence (DF) was measured at a wavelength of $560 \mathrm{~nm}$ for 60 seconds post illumination every hour for five days. Images produced were analysed by IndiGO ${ }^{\mathrm{TM}}$ software, background corrected and detrended. Background corrected data was analysed using BRASS.

\section{Quantitative real-time polymerase chain reaction (qRT-PCR)}

RNA was extracted from $40 \mathrm{mg}$ fresh weight of two-week-old seedlings using a Qiagen (Manchester, UK) 
RNeasy plant mini kit with on-column DNase treatment as per manufacturer's instructions. RNA quality and quantity were measured using a Nanodrop 1000 spectrophotometer (Thermo Scientific, USA). cDNA $(500 \mathrm{ng})$ was produced using Thermo Scientific RevertAid kit with oligo $(\mathrm{dT})_{18}$ primers as per manufacturer's instructions. Primers for qRT-PCR were designed using Primer3 or NCBI Primer-BLAST. A list of qRTPCR primers used is provided (Supplemental Table 2). qRT-PCR was performed with SYBR Green PCR kits (Qiagen) which includes premixed HotStarTaq DNA polymerase, SYBR Green I dye and dNTPs. Samples were analysed in a Qiagen Rotor-Gene Q cycler, and melt curve analysis was also performed. Each experiment was performed independently three times providing three biological replicates, and three technical replicates were performed within each experiment.

\section{RNA sequencing}

For RNA-seq analyses, Col-0, parp1-2, parp2-1 ,parp3-1, parp1-2x2-1x3-1,srt1-4 hete,srt2-1 and srt1-4hete $x$ srt2-1 mutant seedlings were grown for two weeks in the conditions used for the other experiments. Three independent biological replicate seedlings were collected at ZT6 and ZT18 representing the day and night samples.

RNA was extracted using the same method as for the qRT-PCR. RNAseq libraries were generated using the Illumina TruSeq RNA Prep Kit v2 (Illumina, San Diego, CA) and sequenced by BGI-Hongkong (Hong Kong, China) on an Illumina Hiseq 4000 with 100 base pair paired-end reads. The sequencing data were provided demultiplexed, pre-filtered and with the adaptor sequences trimmed off. Quality Checks (QC) on the data were performed using the fastQC software v0.11.4 (https://www.bioinformatics.babraham.ac.uk/projects/fastqc/).

\section{RNA sequencing alignment, quantification and differential expression}

The RNA-Seq reads were aligned against Arabidopis thaliana TAIR10 transcriptome models using bowtie2 (Langmead \& Salzberg, 2012) with the -very-sensitive preset. Transcript quantification was performed by the eXpress software v1.5.1 (Roberts \& Pachter, 2013) using default parameters. The transcript counts were used as input for the R/Bioconductor package baySeq v2.6.0 (Hardcastle \& Kelly, 2010) in order to perform differential expression analysis. This package was run using default parameters and with a false discovery rate (FDR) of 0.05. For each condition (day, night), each mutant was compared with Col-0.

Publicly available datasets from studies of differential expression related to light signalling and a control unrelated to light signalling were downloaded from the European Bioinformatics Institute (EBI) for comparison with the results for our srt1-4 hete mutant. These external datasets are listed in Supplemental Table 3. The differentially expressed transcripts were divided into a list of up regulated and a list of down regulated transcripts. Then the overlap between the up and down regulated lists for srt1-4 hete and each of the corresponding up and down regulated lists for the five comparisons was identified. The R/Bioconductor package, GeneOverlap v1.24.0 (Shen, 2020), was used to determine whether the overlap was statistically significant. Because the internal and external datasets do not contain exactly the same genes, the parameter "genome size" was given as the number of gene identifiers common to both data sets. For the comparison between srt1-4 hete in the day and the "white light" (WL) data from EBI, the genes in each of the four overlaps were tested for enrichment of Gene Ontology (GO) terms (Ashburner et al ., 2000) using the R/Bioconductor package GOstats v2.54.0 (Falcon \& Gentleman, 2007) with GO.db v3.11.4 (Carlson, 2020a) and org.At.tair.db v3.11.4 (Carlson, 2020b).

\section{Other Statistical Analysis}

The Student's t test was used to compare differences between two groups. Where there were more than two groups, a one-way ANOVA was used. A two-way ANOVA was performed in experiments where both genotypes and conditions were tested. Non-parametric alternatives were used when assumptions for parametric tests were not met. Sigmaplot ${ }^{\mathrm{TM}}$ (Systat Software Inc, USA) was used to perform these statistical analyses. Data are presented as the mean plus and minus the standard deviation.

\section{Results}




\section{NADase inhibitors lengthen the period of circadian rhythms}

We confirmed previous reports that nicotinamide increases circadian period in Arabidopsis (Dodd et al 2007; Mombearts et al ., 2019). The period of the circadian rhythms of leaf position of untreated Col-0 was 24.5 $\pm 1.5 \mathrm{~h}$ which increased to $26.00 \pm 0.5 \mathrm{~h}, \mathrm{P}<0.001$ in the presence of $50 \mathrm{mM}$ nicotinamide (Figure 1a,b). Similarly, using CLOCK ASSOCIATED 1 (CCA1) fused to the luciferase reporter gene (CCA1:luc)we measured an increase of circadian period from $23.7 \pm 0.3 \mathrm{~h}$, to $26.0 \pm 0.5 \mathrm{~h}$ following treatment with $50 \mathrm{mM}$ nicotinamide (Figure 1c).

To investigate if PARPs might be mediating the effect of nicotinamide on the circadian oscillator, we measured the response to 3-Methoxybenzamide (3MB), an inhibitor that has been reported to be more specific than nicotinamide for PARP activity (Chen et al ., 1994). 3MB increased the period of circadian oscillations of $C C A 1:: L U C$ luminescence (Figure 2a, untreated $25.2 \pm 0.6 \mathrm{~h}$, DMSO solvent control $25.4 \pm 0.7 \mathrm{~h}, 1 \mathrm{mM}$ $3 \mathrm{MB} 26.0 \pm 0.6 \mathrm{~h}, 2 \mathrm{mM} 3 \mathrm{MB} 27.1 \pm 0.7 \mathrm{~h} \mathrm{P}<0.001$ ) and leaf movement (Figure $2 \mathrm{~b}$; untreated $23.2 \pm 0.7$ h; $3 \mathrm{MB} 23.9 \pm 0.8 \mathrm{~h}, \mathrm{P}=0.012)$.

\section{$P A R P$ mutants do not have altered circadian rhythms}

The sensitivity of circadian rhythms to nicotinamide and 3MB might suggest a role of PARPs in the regulation of the plant circadian oscillator, which prompted us to investigate the regulation of $P A R P$ transcripts and to isolate mutants in the three knownPARP encoding genes in Arabidopsis. None of the transcripts encoding PARPs in Arabidopsis oscillated with a circadian dynamic (Supplemental Figure 1). We were unable to detect PARP3 by qRT-PCR due to low abundance.

Homozygous T-DNA insertion alleles were identified for the parp1-2 (At4G02390; SAIL line 1250B03), parp21 (At2G31320; GABI line 380E06), and parp3-1 (At5G22470; SAIK line 108092) mutants (Supplemental Figure 2a and Supplemental Table 1) These were combined to generate aparp1-2x2-1x3-1 triple mutant (Supplemental Figure 2b) in which we could not detect transcripts of PARP1, 2 or 3 (Supplemental Figure 2c). We found no evidence that PARP activity contributes to circadian regulation as measured by leaf movement analysis (Figure 3a-d) or the measurement of circadian rhythms of delayed chlorophyll fluorescence (DF; Supplemental Figure 3) because circadian periods were indistinguishable from wild type background (leaf movement Col-0 $24.4 \pm 1.0 \mathrm{~h}$, parp $1-225 \pm 1.3 \mathrm{~h}$, Col-0 $25.0 \pm 1.4 \mathrm{~h}$, parp2 $-125.3 \pm 1.1 \mathrm{~h}$, Col-0 24.1

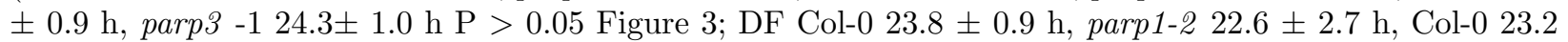

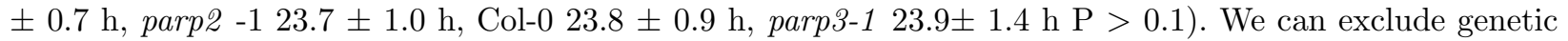
redundancy between the three known PARP-encoding genes because the triple mutant was also without effect on leaf movement (Col-0 $23.8 \pm 1.5 \mathrm{~h}$, parp1-2x2-1x3-1 $24.6 \pm 1.9 \mathrm{~h}, \mathrm{P}=0.190$; Figure 3d) and DF (Col-0 23.5 \pm 0.6 h, parp1-2x2-1x3-1 $24.0 \pm 0.9$ h, P = 0.029; Supplemental Figure 3d).

\section{Loss of SRT1 function causes embryo lethality}

To investigate if sirtuin activity contributes to the response of the circadian oscillator to nicotinamide we identified four different alleles of SRT1 (At5G55760) T-DNA insertion mutants (srt1-1 ; SALK_086287, srt1-2 ;SALK_001493,srt1-3 ;SALK_064336 and srt1-4 ;SAIL_552_E02; Figure 4a and Supplemental Table 1). However, qRT-PCR, demonstrated that three of the lines had little effect on the expression of the SRT1 gene compared to Col-0 (srt1-1, srt1-2 and srt1-3; Figure 4b). For this reason, we proceeded only to investigate the T-DNA insertion mutant srt1-4 (SAIL_552_E02) with the insert located 98 bp from the start of exon 5 which resulted in reduced expression of SRT1(Figure 4a, b). We confirmed that the srt2-1 (At5G09230; SALK_149295; Supplemental Table 1) mutant (previously described in Wanget al ., 2010) has an insert located $38 \mathrm{bp}$ from the start of exon 2 and reduced expression of SRT2 (Figure 4a,b). We were unable to isolate homozygous T-DNA lines of srt1-4 because it caused an embryo lethal phenotype which was not present in heterozygotic mutants (Figure 4c). To confirm embryo lethality is due to the absence ofSRT1, we generated complementation lines with a native promoter and the full length SRT1 gene. All of the complementation lines had normal embryo development, which along with a lack of an embryo phenotype in heterozygotes suggests that it is loss of SIRT1 that affects embryo development, rather than a dominant negative effect of the TDNA insertion (Figure 4c,d). Both SRT1 and SRT2 are highly expressed 
in siliques and flowers which determine embryo development (Supplemental Figure 4). Because full knock out ofSRT1 was embryo lethal, for the rest of the investigation we used heterozygous srt1-4 plants which were knockdowns, having less than half the expression of SRT1 compared to the Col-0 background (Figure 4b).

\section{Sirtuin mutants do not have altered circadian rhythms}

SRT1 and 2 transcript abundance was weakly rhythmic in 24 hours of constant light (Supplemental Figure 1) but we found no evidence that SIRTUINS participate in the regulation of circadian period in Arabidopsis. Neither the srt2-1 knock out, the srt1-4 SRT1 heterozygous knock down or double mutants of those had any significant effect on the circadian period of leaf movement rhythms (Figure 3e,f,g, Col-0 $24.1 \pm 2.2 \mathrm{~h}$, srt1-4 SRT1 $24.7 \pm 2.4 \mathrm{~h}$, srt2-1 $24.0 \pm 1.6 \mathrm{~h}$; srt1-4 SRT1 x 2-1 $24.2 \pm 3.4 \mathrm{~h} \mathrm{P}>0.3)$. Similarly, knock down of SRT1 and knockout of SRT2 was without effect on circadian rhythms of delayed chlorophyll fluorescence (Supplemental Figure 3e,f, Col-0 $24.2 \pm 3.1$ h, srt1-4 hete $24.4 \pm 3.3$ h, srt2-1 24. $\pm 0.6 \mathrm{~h}$ P >0.4).

\section{parg1-1 is a long circadian period mutant}

The lack of effect of $P A R P$ and $S R T$ mutants on circadian period might be unexpected because the $t e j$ 1 mutation of $P A R G 1$, which is predicted to encode a poly(ADPribose) glycohydrolase that counter acts PARP activity has been reported to increase the period of circadian rhythms in Arabidopsis (Panda et al ., 2002). We therefore reinvestigated whether lesions in PARG1 can affect circadian period. As reported previously, tej-1 (a point mutation which results in a change of the glycine at position 262 to a glutamic acid) increases circadian period (Supplemental Figure 5a, C24 $26.8 \pm 0.6 \mathrm{~h}$, tej -1 $30.5 \pm 2.64 \mathrm{~h}, \mathrm{P}=0.006$ ). Having confirmed the phenotype of the tej-1 mutant, we next sought to confirm whether the phenotype of tej-1 was due to a lesion inPARG1. We obtained a second allele of PARG1 (At2g31870), we named as parg1-1 (SALK_147805, NASC; Figure 5a). When transformed with the CCA1::LUC reporter,parg1 -1 mutants had a slightly longer circadian period of luminescence (Supplemental Figure 5b, Col-0 24.5 \pm 0.6 h, parg1 -1 24.8 $\pm 0.4 \mathrm{~h}, \mathrm{P}=0.021$ ). The mean period in circadian rhythms of leaf movement was $1-1.5$ hours longer than wild type (Figure 5b, Col-0 $24.3 \pm 1.0 \mathrm{~h}$, parg1-1 $25.4 \pm 1.3 \mathrm{~h}, \mathrm{P}<0.001$ ). Similarly, circadian rhythms in DF were long period in the parg1-1 mutant (Figure 5c, Col-0 $23.4 \pm 0.9 \mathrm{~h}, \operatorname{parg1-1} 25.0 \pm 1.1 \mathrm{~h}, \mathrm{P}=0.011$ ). We also isolated insertion mutants of PARG2 (At2g31865),parg2-1 (GK072_B04, NASC), to determine if this close gene family member might also contribute to circadian regulation (Figure 5a). The parg2-1 mutant by contrast had weak to no effect on circadian rhythms of leaf movement (Figure 5d, Col-0 $24.5 \pm 0.7 \mathrm{~h}$, parg2-124.8 $\pm 0.6 \mathrm{~h}, \mathrm{P}=0.209$ ) or delayed chlorophyll fluorescence (Figure 5e, Col-0 $24.23 \pm 3.06 \mathrm{~h}$, parg2 $-123.2 \pm 0.8 \mathrm{~h}, \mathrm{P}=0.667)$. Thus, the effects of the PARG1 mutant are specific to that member of the gene family.

\section{Nicotinamide acts through a pathway independent of PARP1, 2 and 3, PARG2, and SIRTUIN} 1 and 2

We have previously proposed that nicotinamide regulates circadian period in Arabidopsis through inhibition of ADPRcyclase activity (Dodd et al., 2007). Here, we tested a counter hypothesis that nicotinamide regulates circadian clocks through the inhibition of PARP or SRT activity. We reasoned that in knock downs of PARP or $S R T$ that nicotinamide would be less effective if its mode of action in the circadian system was associated with the activity of the encoded proteins. We found no evidence that PARPs or sirtuins are the target for nicotinamide to affect circadian rhythms. The period of circadian rhythms of leaf movement in the parp1-2 , parp2-1 andparp3-1 single mutants was longer in the presence of nicotinamide in comparison to untreated controls (Figure 6 a,b,c; circadian period estimates for each genotype minus and plus $50 \mathrm{mM}$ nicotinamide follow:parp1-2 $24.8 \pm 2.1,25.3 \pm 2.4 \mathrm{~h}, \mathrm{P}=0.002$; parp2-1 $25.0 \pm 0.8 \mathrm{~h}, 26.1 \pm 1.1 \mathrm{~h}, \mathrm{P}=0.007$; parp3-1 $24.5 \pm 2.1 \mathrm{~h}, 25.7 \pm 0.8 \mathrm{~h}, \mathrm{P}=0.001)$. Circadian rhythms of leaf movement in theparp1-2x2-1x3-1 mutant also had a long period with the addition of nicotinamide (Figure 6d; parp1-2x2-1x3-1 25.5 $\pm 1.0 \mathrm{~h}$,parp1-2x21x3-1 $50 \mathrm{mM}$ nicotinamide $26.8 \pm 1.2 \mathrm{~h}$, two-way ANOVA, $\left.\mathrm{P}_{\text {(genotype) }}=0.146, \mathrm{P}_{\text {(treatment) }}<0.001\right)$. We also confirmed the lack of interaction between PARPs and nicotinamide by examining the circadian regulation of transcript abundance (Figure 7). Nicotinamide treatment delayed the peak time of CCA1, PRR 7 and TOC1 
expression by 4 hours in both Col- 0 and parp1-2x2-1x3-1 with no difference between the genotypes $(\mathrm{P}>$ $0.2)$.

Sirtuins also are not required for the response to nicotinamide.srt1-4 hete and srt2-1 mutants had a significantly longer period in the presence of nicotinamide (Figure 6e,f,g circadian period estimates for each genotype minus and plus $50 \mathrm{mM}$ nicotinamide follow:srt1-4 hete $24.7 \pm 0.9 \mathrm{~h}$, srt1-4 hete $25.7 \pm 0.8 \mathrm{~h}$, two-way ANOVA, $\mathrm{P}_{\text {(genotype) }}=0.521, \mathrm{P}_{\text {(treatment) }}=0.05 ;$ srt2-1 $24.8 \pm 1.3 \mathrm{~h}, 25.5 \pm 1.7 \mathrm{~h}$, two-way ANO-

$\mathrm{VA}, \mathrm{P}_{\text {(genotype) }}=0.717, \mathrm{P}_{\text {(treatment) }}=0.002 ;$ srt1-4 hetex2-1 $24.2 \pm 0.7 \mathrm{~h}, 25.0 \pm 0.5 \mathrm{~h}$, two-way ANOVA $\left.\mathrm{P}_{(\text {genotype })}=0.9, \mathrm{P}_{(\text {treatment })}=0.013\right)$.

We also examined the effects of nicotinamide in the PARG mutants, whilst these are not expected to be targets, there is a possibility of an interaction because PARG reverses the effects of PARP. Nicotinamide treatment lengthens the period of circadian rhythms of leaf movement inparg1-1 mutants (Figure 6h, parg1-1 $25.37 \pm 1.5 \mathrm{~h}, \operatorname{parg} 1-1+$ nicotinamide $26.59 \pm 1.2 \mathrm{~h}, \mathrm{P}<0.001$ ) andparg2-1 (figure 6i, parg2-1 $25.00 \pm 1.7$ $\mathrm{h}$, parg2-1 + nicotinamide $26.1 \pm 1.1 \mathrm{~h}, \mathrm{P}<0.001$ ). Taken together we found no evidence that nicotinamide regulates the circadian period of Arabidopsis through the action of PARPs, PARGs or sirtuins.

\section{PARP regulates flowering time in Arabidopsis by modulating $F L C$ expression}

Since we found no evidence that PARPs and SRTs are implicated in circadian regulation, we performed RNAseq to investigate their wider role. We performed these measurements under $12 \mathrm{~h} \mathrm{~L} / 12 \mathrm{~h} \mathrm{D}$ light cycles, sampling in the middle of the day (ZT 6) and night (ZT18) to capture temporal regulation of gene expression. In parp mutants, only 11 genes were mis-regulated in all the parp mutants in the day (number of mis-regulated genes: parp1-2 day 27, night 57,parp2-1 day 43, night 39, parp3-1 day 79, night 44 and parp12x2-1x3-1 day227, night 41) (Figure 8 a, b and Supplemental Table 4). To attempt to confirm RNAseq data, we performed qRT-PCR to measure the transcript abundance of several genes that were found to have similar changes in all the parp lines. Genes were chosen representing no change (PRR7), a decrease (GP2, SUS4 ), a moderate increase (CCA1) or a large increase $(A H A 8)$ in abundance. The independent qRT-PCR experiments were consistent with the effects measured by RNAseq (Supplemental figure 6).

We were surprised that so few genes were differentially expressed in thePARP mutants and therefore we tested for any physiological consequences of the change in expression of the few genes we detected. We focused on $F L C$, because this was significantly upregulated in all PARP mutants in the day and night, except parp3-1 at night where the transcript counts were higher but the FDR did not reach statistical significance $(\mathrm{FDR}=0.14)$. It is $P A R P 1$ and 2that are expressed in vegetative tissue (Supplemental Figure 1) which might explain why parp3-1 had a less effect on FLC abundance than mutations in PARP1 and 2 . First, we confirmed that FLC is differentially expressed in PARPmutants by qRT-PCR of independent samples of the mutant plants (Figure $8 \mathrm{c}, \mathrm{d}$ ). These data are strongly indicative that PARP activity can regulate $F L C$ gene expression. We found that the effects on FLC might be meaningful because the elevation of the flowering suppressor was associated with a delay in the days to flowering and an increase of the number of leaves at flowering in the parpmutants, and this was specific to short days (Figure 8e,f). The flowering phenotype of the parp mutants is therefore consistent with the molecular phenotype as identified by the RNAseq, supporting the conclusion that PARP activity is not a major regulator of gene expression, at least in stress-free conditions, but can affect $F L C$ expression and flowering time.

\section{Sirtuin 1 affects gene expression during the day}

In contrast to the PARPs, alterations to sirtuin expression had a profound effect on the transcriptome. In srt1-4 heterozygous plants, 6490 genes were differentially expressed in the day (sampling at ZT 6) compared to Col-0 (Figure 9a and Supplemental Table 5). This effect was mostly specific to the day, because at night the transcripts of only 49 genes were differently expressed (sampling at ZT 18) (Figure 9a and Supplemental Table 5). Our results suggest that thesirtuin1 might have a very important role in day-time gene expression. To further investigate this hypothesis we compared our transcription profiles with publicly-available light signalling- and sucrose-related transcriptomes (white light, red light, UV-B and sucrose; Supplemental Table 3) and a control transcriptome unrelated to light signalling (heat \& salt) downloaded from the EBI (htt- 
ps://www.ebi.ac.uk/gxa/). There were highly significant overlaps between SRT1 -regulated transcript sets and those regulated by light and sucrose, withSRT1 expression affecting the gene expression in the opposite direction to light and sucrose signals (Figure 9b, Supplemental Table 6, 7). A wide range of GO terms were associated with day and SRT1 regulation many of which can be associated with light signalling and growth (Supplemental Table 7). These data indicated that SRT1 might positively regulate transcripts regulated in the day by light signalling and light induction of sugar production by photosynthesis. By contrast srt2-1 had a relatively small effect on gene expression, with only 235 transcripts at day and 97 at night being differentially expressed (Figure 9a and Supplemental Table 5). The dramatic effect of reduced SRT1 expression on transcript abundance was confirmed by analysis in a srt1-4 hete $x$ srt2-1 which had an overlap of 4143 differentially expressed transcripts with the srt1-4 hete single mutant (Figure 9a).

To confirm the results of the RNAseq data we performed the experiment again three times with new biological material for each experiment and a selected subset of genes to be measured by qRT-PCR to compare to the findings of the RNAseq. At2G36970 (UDP-Glycosyltransferase superfamily protein) was selected because it has a large decrease in abundance in RNAseq in the mutant. AT5G55580 (Mitochondrial transcription termination factor family protein) and AT5G55540 (were selected to represent transcripts that had smaller changes in abundance. AT5G62720 (tornado 1), AT4G11830 (phospholipase D gamma 2) and AT2G15880 (Leucine-rich repeat (LRR) family protein) were selected representing large increases in abundance changes in srt1-4 hete in the RNAseq data (Supplemental table 5 and Supplemental Figure 7). The qRT-PCR independently confirmed the RNAseq experiment findings about the effects on gene expression during the day, showing that AT2G36970 , At5G55540 and AT5G55580 had reduced expression and AT5G62720, $A T 4 G 11830$ and AT2G15880 were increased in expression in the srt1-4 mutant during the day (Supplemental Figure 7a and b). Similarly, the qRT-PCR confirmed the findings of the effects on gene expression that we measured at night with RNAseq, demonstrating reduced expression of AT2G36970,AT5G55580, At5G55540, AT5G62720 and increased expression at night of AT4G11830 and AT2G15880 in srt1-4 (Supplemental Figure 7c and d). We also examined the abundance of these transcripts by qRT-PCR in two SRT1 artificial microRNA lines, which also knocked down SRT1 expression (Supplemental Figure 8a). The microRNA lines were not quite as effective in knocking down srt1 expression assrt1-4 hete (Supplemental Figure 8a). All the transcripts had similar patterns of abundance between srt1-4 hete and SRTmicroRNA lines in the day, though in line with their reduced effect onsrt1 expression, compared to srt1-4, the microRNA lines had less of an effect on the abundance of the transcripts (Supplemental Figure 7a). The trend of similar but reduced effects of the microRNA lines compared to srt1-4 was also found at night (Supplemental Figure 7c). For AT2G36970 , AT5G55580, AT5G62720 expression was reduced in srt1-4 and in the microRNA lines, and for AT4G11830 and AT2G15880 expression was increased insrt1-4 and the microRNA lines (Supplemental Figure 7c). The exception being AT5G5540, for which at night there was little effect of the microRNA lines, but a strong effect of srt1-4. Thus, in independent experiments we confirmed that srt1-4 and micro RNA lines had similar effects on transcript abundance and the degree of effect was in line with the abundance of the srt 1 transcript. This demonstrates that the effects on the transcriptome are due to changes in SRT1 expression.

Because we found profound effects of SRT1 expression in the light, and a strong overlap with genes that are regulated by light, either directly by signalling, or potentially indirectly by sugars we also performed qRT-PCR to measure light signalling gene expression in RNA extracted from SRT1 artificial microRNA linesandsrt1-1 , srt1-2 , srt1-3, srt1-4 hete andsrt2-1 mutants (Supplemental figure 8a). We chose several light signalling related genes (AT2G37678, FHY1 far-red elongated hypocotyl 1, AT5G02200 FHL far-red elongated hypocotyl 1 like, AT2G42870 PAR1 phy rapidly regulated 1, AT1G70290 TPS8 trehalose-6-phosphatase synthase 8 ). There was good correspondence between the effects of srt1-4 hete and SRT1 microRNA lines on the expression of these light-regulated signalling transcripts (Supplemental Figure 8b).srt1-4 and micro RNA lines increased the expression of all of the selected light signalling genes confirming that SRT1 affects their abundance (Supplemental figure 8b), as had been found in the RNAseq (Supplemental Figure 8c)..

\section{Discussion}


Understanding of the mechanisms which regulate circadian timing has been enhanced greatly in the past few decades, with the discovery of post-transcriptional and post-translational modifications, chromatin remodelling and cytosolic events. We have been investigating how cellular metabolism, an important output of the circadian clock, might reciprocally communicate with the circadian clock to modify circadian clock behaviour. To this end, we screened a group of $\mathrm{NAD}^{+}$-related mutants for circadian phenotypes. This study was motivated by the extensive literature concerning the function of the PARPs and SRT in regulating the circadian clock of mammals and the effect of nicotinamide being common between the plant and mammalian systems (Dodd et al ., 2007; Nakahata et al ., 2008; Nakahata et al ., 2009; Asher et al ., 2010). Here, we found no evidence that mutation of the PARPs or SRT affected circadian period, the response to nicotinamide or affected specifically the expression of circadian oscillator genes. Our finding that the effects of nicotinamide on the circadian oscillator of plants are not associated with inhibition of the PARP and SRT NADases means that the mechanisms by which NAD regulates the circadian clock of Arabidopsis might be different to that which occur in mammals (Dodd et al ., 2007; Nakahata et al ., 2008; Nakahata et al ., 2009; Asheret al ., 2010).. We cannot exclude the possibility that unidentified members of the PARP and $S R T$ gene families could be targets for nicotinamide. Based on these findings and our previous data we favour alternative explanations for the effects of nicotinamide on the circadian oscillator. Nicotinamide is an inhibitor also of ADPRcyclase which generates the $\mathrm{Ca}^{2+}$ agonist, cADPR (Abdul-Awal et al 2016). and we have measured circadian oscillations of cADPR in Arabidopsis (Dodd et al ., 2007) Furthermore, we reported that $\mathrm{Ca}^{2+}$ affects circadian period through regulation of TOC1 by CALMODULIN-LIKE 24 (CML24) (Martí Ruiz et al ., 2018). $\mathrm{Ca}^{2+}$ is sensed by the circadian oscillator by CML24 and the effects of mutations inCML24 on circadian period are not additive to the effects of nicotinamide, which is consistent with the effect of nicotinamide being to abolish the $\mathrm{Ca}^{2+}$ signal through inhibition of ADPR cyclase activity (Martí Ruiz et al ., 2018). However nicotinamide has a greater effect on circadian period than mutation of CML24alone, suggesting that either there are other $\mathrm{Ca}^{2+}$ sensors in the circadian system or nicotinamide has other targets, such as reduction of H3K4me3 accumulation (Malapeira et al ., 2012) and the action of BIG (Hearn et al ., 2018). TOR seems to be required for the response of the circadian oscillator to nicotinamide, and it has been proposed this might be due to altered energy production by the mitochondria (Zhang et al., 2019). The mitochondria are affected by and regulate $\mathrm{Ca}^{2+}$ dynamics and therefore a dual role for TOR and $\mathrm{Ca}^{2+}$ signalling in regulation of the circadian oscillator due to mitochondrial responses to nicotinamide is plausible (Bravo-Sagua et al., 2017).

We also found a PARP inhibitor 3MB increases circadian period similarly to nicotinamide (Figure 2). However, 3MB is a structural analogue of nicotinamide, and therefore may also target enzymes other than PARP which bind $\mathrm{NAD}^{+}$or nicotinamide. In contrast, the use of another chemical inhibitor of PARP activity, thymidine, did not affect the period length or amplitude of circadian rhythms of CCA1::LUC bioluminescence (Malapeira et al ., 2012). Overall, based on these observations there is no strong evidence for the role of PARPs in mediating the effect of nicotinamide on clock function in Arabidopsis, and the specificity of the drugs used is questionable, as different drugs targeting PARPs have conflicting effects on circadian period. Whilst PARPs do not affect the circadian oscillator, we found evidence that they affect seasonal timing through the regulation of $F L C$ expression and flowering time.

The lack of a role for the PARPs is at first sight surprising given the reproducible role of PARG1 in setting circadian period (Figure 5). Panda et al ., (2002) predicted that parp mutants would have reduced circadian period due to the period reducing effect of the PARP inhibitor $3 \mathrm{AB}$, however our data indicate that the chemical inhibition of PARP may have multiple effects and may act independently of PARP. These results also lead us to speculate how PARGs could specifically modulate circadian clock function without the apparent reciprocal effect seen in parp mutants. It is possible that other proteins encoded in the Arabidopsis genome which have ADP-ribosyl transferase activity might be responsible for counteracting PARGs. For example, the SRO (SIMILAR TO RCD-ONE) family of plant-specific proteins have the conserved PARP catalytic domain, however, these have been found not to possess ADP-ribosyl transferase activity (Jaspers et al ., 2010).

We also found no evidence for a role of sirtuins in modulating clock behaviour in Arabidopsis. Neither srt 
single nor double mutants had significantly different circadian periods to wild type in assays of leaf movement or DF (Figure 3 and Supplemental Figure 3). This suggests that unlike mammals, sirtuins are not regulators of the circadian oscillator in Arabidopsis. Sirtuins are intimately associated with central circadian clock components in mice (Asher et al ., 2008; Chang \& Guarente, 2013; Nakahata et al ., 2008; Nakahata et al ., 2009). In mammals, there is significant evidence that aspects of nicotinamide effects might be mediated through sirtuins, due to the long period of rhythms in locomotor activity seen in brain-specificsirt1 mutants (Chang \& Guarente, 2013). Furthermore, resveratrol, an activator of SIRT1 (Lagouge et al ., 2006), causes a shortened period of circadian rhythms in locomotor activity of grey mouse lemur (Das et al ., 2010; Pifferi et al ., 2011) and increased circadian clock gene expression in Rat-1 fibroblast cells (Oike \& Kobori, 2008). In Arabidopsis, acetylation has been established as a regulatory mechanism controlling circadian clock gene expression (Farinas \& Mas, 2011; Malapeira et al ., 2012; Song \& Noh, 2012), and flowering time by modifying chromatin acetylation at the $F L C$ locus (Ausin et al ., 2004; He et al ., 2003; Kimet al ., 2004; Xiao et al $., 2013)$. As acetylation is evidently involved within the circadian clock in Arabidopsis at a transcriptional level (Farinas \& Mas, 2011; Malapeira et al ., 2012; Perales \& Mas, 2007), other non-NAD ${ }^{+}$-dependent deacetylases must be responsible. A corepressor protein TOPLESS (TPL) has been found to interact with PRR7, 9 and 5 at the promoter regions of $C C A 1$ and $L H Y$ to repress transcription (Wang et al ., 2013). This was found to require histone deacetylase activity; treatment with Trichostatin A (TSA) disrupts this repression, and histone deacetylase 6 (HDA6) forms a complex with TPL and PRR9 in vitro (Wang et al ., 2013).

Further evidence that the effect of nicotinamide is independent of sirtuin-like activity is provided by the finding that H3K56ac was decreased in nicotinamide treated plants, opposite to the expectation if deactylation is a nicotinamide-sensitive activity (Malapeira et al ., 2012). C646, an acetylase inhibitor, phenocopies the effects of nicotinamide also suggesting that sirtuin-like deactylase activity is not a regulator of the Arabidopsis circadian oscillator (Malapeiraet al ., 2012). Interestingly, nicotinamide treatment increases histone acetylation at the VIN3 locus, and induces FLCrepression and flowering (Bond et al ., 2009). However, VIN3 expression was not altered in sirtuin mutants, which suggested the effect of nicotinamide on VIN3 expression also was not mediated by sirtuins.

We found profound effects of SRT1 on transcript abundance only during the day (Figure 9). Knockdown of srt1 affected the abundance of nearly one third of the transcriptome in the day but not at night. The transcripts affected were strongly associated with light and sugar signalling pathways, and less so with those associated with heat and salt stress (Figure 9). This was confirmed in microRNA lines and by using qRT-PCR in independent experiments. Our finding that SRT1 can have such profound effects on the transcriptome in the day and that complete knock out of SRT1 is embryo lethal has not been reported in previous studies, possibly because other investigations have focused on lines with less strong effects on SRT1 expression. Liuet al ., (2017) found that the T-DNA insertion mutant linesrt1-2 (SALK_001493) has weak effects on SRT1 expression and that RNAi lines had stronger effects on SRT1 expression and metabolism. From RNAi and over expression studies Liu et al., (2017) concluded that SRT1 interacts with Arabidopsis cMyc-Binding Protein 1 (AtMBP-1), which is a transcriptional repressor to regulate AtMBP-1 targets resulting in altered gene expression and metabolism. We also found that srt1-2 has weak or no effects on SRT1 expression, and that RNAi was more effective in reducing SRT1 transcript abundance (Figure 4b; Supplemental Figure 8a). Zhang et al ., (2018) reported that srt1-1 and srt1-2 completely abolished SRT1 expression resulting in phenotypes associated with ethylene responses. The qPCR primers used by Zhang et al ., (2018) were down stream of the srt1-2 T-DNA insert representing the end of the CDS, whereas we used primers upstream of the srt1-4insertion site in the deacetylase domain and Liu et al., (2017) used primers in the same domain downstream of the location of thesrt1-4 insert (Figure 4A). Taking these together it is clear that the srt1-1 and srt1-2 mutants make transcripts encoding an intact deacetylase domain, which might explain why in our hands and those of Liu et al., 2017 the srt1-1 and srt1-2mutants had weak or no phenotypes. Furthermore, we found that complete knock out of SRT1 by insertion of a T-DNA in the acetylase domain in the srt1-4 mutants was embryo lethal, which was not reported by Zhang et al., (2018) for srt1-1 and srt1-2, which suggests the lines used in the study of Zhang et al.,(2018) were not abolished in SRT1 function. Based 
on our findings that srt1-4 homozygous plants are embryo lethal and thatsrt1-4 heterozygous plants have lower expression of SRT1 than RNAi lines and other T-DNA alleles, we conclude that the full extent of the effects of SRT1 have been obscured previously by investigation in lines which have little or no effect on the expression of the SRT1 deacetylase domain (srt1-1, srt1-2), and possibly weaker RNAi knock down lines. The profound regulation of gene expression we find in srt1-4 heterozygous lines and the associated embryo lethal effects of the srt1-4 homozygous lines is associated with the loss of transcripts encoding the deacetylase domain.

The strong correspondence between the transcripts mis-regulated in plants with reduced SRT1 and those regulated by light and sugars (Figure 9) and the opposite direction of the transcript regulation bysrt1-4 to the regulation by light signals (Figure 9) could suggest that SRT1 participates in the regulation of transcripts by pathways activated by light signalling and the light regulation of photosynthesis. Our data suggest that SRT1 is required for the correct regulation of gene expression during the day but independent of a function in the circadian oscillator.

Figure legends

Figure 1. Nicotinamide lengthens the period of circadian rhythms

(a) Mean Y pixel positions of leaves of Col-0 in constant light $\left(60-80 \mu \mathrm{mol} \mathrm{m}^{-1} \mathrm{~s}^{-1}\right)$ with or without two $50 \mathrm{mM}$ nicotinamide treatments one and two days before imaging. (b) Individual FFT-NLLS period estimates and RAE values for circadian rhythms of leaf movement, data combined from separate experiments, untreated $\mathrm{n}_{\text {rhy }}=218$, nicotinamide treated $\mathrm{n}_{\text {rhy }}=292(\mathrm{c})$ Rhythms of CCA1:LUC luminescence in constant light. Seedling clusters were dosed with $50 \mu \mathrm{l} 5 \mathrm{mM}$ luciferin plus or minus nicotinamide every 12 hours, The points represent mean of $\mathrm{n}_{\text {rhy }}=4$ for each treatment. Error bars represent standard deviation. $\mathrm{N}_{\text {rhy }}=$ the number of rhythmic plants as estimated by FFT NLS

Figure 2. 3MB is a modifier of circadian clock period

(a) 3MB treatment applied in the media lengthens the period of CCA1:LUC rhythms in Col-0 seeds in constant light. Untreated, DMSO and $2 \mathrm{mM} 3 \mathrm{MB} \mathrm{n}=10,1 \mathrm{mM} 3 \mathrm{MB} \mathrm{n}=20$. (b) mean Y pixel positions of leaves of Col-0 seedlings sown onto 0.5 MS media containing DMSO or $0.2 \mathrm{mM} 3 \mathrm{MB}$ and entrained in 12L/12D for 10 - 12 days before transfer into constant light $\left(60-80 \mu \mathrm{mol} \mathrm{m}^{-1} \mathrm{~s}^{-1}\right)$ for imaging Col-0 (untreated $\left.\mathrm{n}_{\mathrm{rhy}}=20,3 \mathrm{MB} \mathrm{n}_{\mathrm{rhy}}=20\right)$ Error bars represent standard deviation.

Figure 3. PARP and SRT mutations do not affect the circadian period of leaf movement.

Circadian rhythms of leaf movement in constant light (LL) of parpand sirtuin mutants from a single representative experiment and individual FFT-NLLS period estimates and R.A.E values from combined separate experiments. Col-0 was used as same control for e and g. (a) Col- $0 \mathrm{n}_{\text {rhy }}=87, \operatorname{parp} 1-2 \mathrm{n}_{\text {rhy }}=75$ (b) Col-0 $\mathrm{n}_{\text {rhy }}=92$, parp2 $-1 \mathrm{n}_{\text {rhy }}=94$ (c) Col- $0 \mathrm{n}_{\text {rhy }}=50$, parp3 $-1 \mathrm{n}_{\text {rhy }}=53$ (d) Col- $0 \mathrm{n}_{\text {rhy }}=63$, parp1-2x2-1x3-1 $\mathrm{n}_{\text {rhy }}=54(\mathrm{e})$ Col- $0 \mathrm{n}_{\text {rhy }}=18$, srt1-4 hete $\mathrm{n}_{\text {rhy }}=33$ (f) Col-0 $\mathrm{n}_{\text {rhy }}=76$, srt2- $1 \mathrm{n}_{\text {rhy }}=105$ (g) Col- $0 \mathrm{n}_{\text {rhy }}$ $=18$, srt $1-4$ hetex $2-1 \mathrm{n}_{\text {rhy }}=19$. Error bars represent standard deviation. $\mathrm{N}_{\text {rhy }}=$ the number of rhythmic plants as estimated by FFT NLS.

Figure 4. srt1-4 insertion line has an embryo lethal phenotype.

(a) Gene structures of SRT1 and SRT2. Confirmed insertion sites are indicated. Positions of relevant primers pairs are indicated. Gene F/R (black arrows) = primers positioned to flank insertion site, qPCR (green arrows $)=$ primers positioned downstream of insertion site. Orange arrows indicate qPCR primers used by Liu et al., (2017) and red arrows indicate qPCR primers used in the study of Zhang et al., (2018) (b) Relative gene expression of SRT1 in srt1-1, srt1-2, srt1-3, srt1-4 hete and srt2-1 measured by qRT-PCR. N = 3 (c) Seeds of Arabidopsis SRT1 mutant alleles and Col- 0 background. srt1-4 heterozygous mutant has aborted seeds (white arrows) that are not observed in Col-0 background or srt1-2 , srt2-1, srt1-2x2-1 and srt1-3x2-1 . White bar indicates $1 \mathrm{~mm}$. (d) Percentage seed set in srt1 mutants and threesrt1-4 complementation lines $(\mathrm{COM}) .(\mathrm{Col}-0 \mathrm{n}=516$, srt1 $1 \mathrm{n}=542$, srt1-2 $\mathrm{n}=509$, srt1-3 $\mathrm{n}=501$, srt1-4 hete $\mathrm{n}=322$, srt1-4 
Complementation line \#1 $\mathrm{n}=602$,srt1-4 Complementation line \#2 $\mathrm{n}=609$ and srt1-4Complementation line \#3 $\mathrm{n}=586)$.

Figure 5. parg 1-1 lengthens circadian period.

(a) Gene structures and confirmed insertion sites of PARG1, withparg1 -1 (SALK_147805) allele, PARG2 and parg2 -1 (GK072_B04). Positions of relevant primers pairs are indicated. Gene F/R (black arrows) = primers positioned to flank insertion site, qPCR (green arrows) = primers positioned downstream of insertion site. (b-e) Circadian rhythms in constant light of leaf movement and delayed chlorophyll fluorescence in parg1-1 (b, c), parg2-1 (d, e) and Col-0 background. (b, d) Mean Y pixel positions of leaf movement from a single representative experiment and individual FFT-NLLS period estimates and R.A.E values. (c, e) Delayed chlorophyll fluorescence. Data are combined from separate experiments, (a) Col- $0 \mathrm{n}_{\mathrm{rhy}}=158$, parg1 $-1 \mathrm{n}_{\text {rhy }}=160$ (b) Col-0 $\mathrm{n}_{\text {rhy }}=10$, parg1- $1 \mathrm{n}_{\text {rhy }}=9$. (c) Col-0 $\mathrm{n}_{\text {rhy }}=83, \operatorname{parg} 2-1 \mathrm{n}_{\text {rhy }}=84$ (d) Col-0 $\mathrm{n}_{\text {rhy }}$ $=12$,parg $1-1 \mathrm{n}_{\text {rhy }}=11$. Error bars represent standard deviation. $\mathrm{N}_{\text {rhy }}=$ the number of rhythmic plants as estimated by FFT NLS.

Figure 6. Nicotinamide increases the period of circadian rhythms of leaf movement in parp, srt and parg mutants.

Circadian period of leaf movement in constant light in Col-0 and mutant lines with and without dosing with $50 \mathrm{mM}$ nicotinamide (nam) one and two days before the start of imaging. Col-0 background minus (white) or plus nicotinamide (grey). PARP mutants are blue, sirtuin mutants are red and PARG mutants are green. Within each plot the darker colour shade represents the mutant line treated with nicotinamide. All data are shown with the mean indicated by a line. (a) Col- $0 \mathrm{n}_{\text {rhy }}=25$, parp $1-2 \mathrm{n}_{\text {rhy }}=22$, Col- $0+$ nic $\mathrm{n}_{\text {rhy }}=12$, parp1-2 + nic $\mathrm{n}_{\text {rhy }}=27$ (b) Col-0 $\mathrm{n}_{\text {rhy }}=22$, parp2-1 $\mathrm{n}_{\text {rhy }}=11$, Col-0 + nic $\mathrm{n}_{\text {rhy }}=25, \operatorname{arp} 1-2+$ nic $\mathrm{n}_{\text {rhy }}$ $=20$ (c) Col-0 (as for (a))parp3-1 $\mathrm{n}_{\text {rhy }}=27$, parp3-1 + nic $\mathrm{n}_{\text {rhy }}=14$ (d) Col-0 $\mathrm{n}_{\text {rhy }}=16$,parp1-2x2-1x3-1 $\mathrm{n}_{\text {rhy }}=9$, Col-0 + nic $\mathrm{n}_{\text {rhy }}=23$, parp1-2x2-1x3-1 + nic $\mathrm{n}_{\text {rhy }}=22\left(\right.$ e) Col- $0 \mathrm{n}_{\text {rhy }}=12$, srt1-4 hete $\mathrm{n}_{\text {rhy }}=33$, Col-0 + nic $\mathrm{n}_{\text {rhy }}=10$, srt1-4 hete + nic $\mathrm{n}_{\text {rhy }}=21$ (f) Col-0 $\mathrm{n}_{\text {rhy }}=15$, srt2- $1 \mathrm{n}_{\text {rhy }}=14$, Col- $0+$ nic $\mathrm{n}_{\text {rhy }}=$ 17, srt2-1 + nic $\mathrm{n}_{\mathrm{rhy}}=18(\mathrm{~g})$ Col-0 (as for (e)), srt1-4 hete $\mathrm{n}_{\mathrm{rhy}}=19$, srt1-4 hete + nic $\mathrm{n}_{\mathrm{rhy}}=29$ (h) parg2-1 $\mathrm{n}_{\text {rhy }}=31, \operatorname{parg} 2-1+$ nic $\mathrm{n}_{\text {rhy }}=41$ (i) Col-0 (as for (h)), Col- $0 \mathrm{n}_{\text {rhy }}=48, \operatorname{parg} 1-1 \mathrm{n}_{\text {rhy }}=22$, Col- $0+$ nic $\mathrm{n}_{\text {rhy }}=36, \operatorname{parg} 1-1+$ nic $\mathrm{n}_{\text {rhy }}=21 . \mathrm{N}_{\text {rhy }}=$ the number of rhythmic plants as estimated by FFT NLS.

Figure 7. Circadian rhythms of circadian oscillator gene expression in parp1-2x2-1x3-1 mutants are delayed in the presence of nicotinamide.

Plants were entrained for nine days in $12 \mathrm{~L} / 12 \mathrm{D}$ before transfer into constant white light. On day seven, plants were transferred onto treatment media (10 $\mathrm{mM}$ nicotinamide or untreated control). Tissue samples (three biological replicates) were harvested every four hours in the third cycle of constant light. Abundance measured using qRT-PCR was normalised to UBQ10 . CCA1, TOC1 and PRR7peak abundance is delayed by nicotinamide treatment in both genotypes. Error bars represent standard deviation.

Figure 8. FLC is misexpressed in parp mutants, which have delayed flowering in short day photoperiods

(a, b) Venn diagrams of the number of transcripts differentially expressed (FDR $<0.05$ ) as measured by RNAseq inparp1-2 , parp2-1 , parp3-1 andparp1-2x2-1 33-1 mutants compared to Col-0 background in (a) day at ZT6 and (b) night at ZT18. (c ,d) Transcript abundance of FLCmeasured by qRT-PCR in Col0, parp1-2, parp2-1 , parp3-1 and parp1-2x2-1x3-1 in the (c) day and (d) night. qRT-PCR normalised to UBQ10 . Seedlings were grown under $12 \mathrm{~h} \mathrm{~L} / 12 \mathrm{~h} \mathrm{D}$ in white light on $1 / 2 \mathrm{MS}$ agar plates for two weeks and sampled during the day (ZT6) and night (ZT18). Growth, harvesting, RNA extraction and qRTPCR analysis for $F L C$ was performed independently of the RNAseq experiments. Error bars represent the standard deviation. $\mathrm{n}=3$. (e, f) Flowering time as measured by the emergence of bolt and numbers of rosette leaves at bolting of Col-0, andparp1-2, parp2-1, parp3-1, parp1-2x2-1x3-1 under (e) long (16 h L:8 h $\mathrm{D})$ or (f) short day $(8 \mathrm{~h} \mathrm{~L}: 16 \mathrm{~h} \mathrm{D})$. The number of rosette leaves and bolting time were recorded when the emerging bolt was $5 \mathrm{~mm}$ high. Dots represent the individual plants and the black horizontal bars the mean. 
In long photoperiods $n=12$ for each genotype and in short photoperiods $n=15$ for each genotype. The Kruskal-Wallis-one-way analysis of variance was used to determine if there was any overall difference among the 5 genotypes. Dunn's method was then used to test the significance of pairwise comparisons between Col-0 and each mutant. Single asterisks indicate $P$ [?] 0.05. Col-0 (white) parp1-2(yellow), parp2-1 (red), parp3-1 (green) and parp1-2x2-1x3-1 (blue)

Figure 9. srt1-4 hete affects the accumulation of transcripts regulated in the day

Reduced expression of SRT1 in a srt1-4 heterozygous mutant has a profound effect on the abundance of transcripts in the day. (a) Venn diagram showing the number of differentially expressed genes (FDR $<0.05)$ in srt1-4 hete, srt2-1 andsrt1-4hete $x$ 2-1 mutants compared to Col-0 background in the day and the night as measured by RNAseq. Lists of differently expressed genes are given in Supplemental Table 5. (b) Venn diagrams showing the overlap between lists of up and down regulated genes in thesrt1-4 hete mutant at ZT6 and in the "white light" transcriptome from the EBI Gene Expression Atlas (Supplemental Table 3). There is a high degree of correspondence between transcripts up regulated in srt1-4 hete in the day and transcripts that are down regulated in a white light treatment

. Similarly, transcripts that are up regulated by whte lighIt are often in lower abundance in the mutant. (c)

The number of differentially expressed genes in common between thesrt1- 4 heterozygous mutants and plants treated with a particular treatments of white, red light and UV-A light, $1 \%$ sucrose or combined heat and salt stress, expressed as a percentage of the total number of differentially expressed genes in srt1-4 heterozygous mutants (see methods and Supplemental Table 3 for the data sets used). The differentially expressed genes in the srt1-4 heterozygous mutants are listed in Supplemental Table 5.

\section{Supplemental Figures}

\section{Supplemental figure 1 PARP, PARG and sirtuin transcript abundance in continuous light.}

Plants were entrained for nine days in $12 \mathrm{~L} / 12 \mathrm{D}$ before transfer into constant white light. Tissue samples (three biological replicates) were harvested every four hours in the third cycle of constant light. Transcript abundance as measured by qRT-PCR was normalised to $U B Q 10$. Bar indicates \pm the standard deviation. The light and grey bars indicate subjective day and subjective night, respectively.

\section{Supplemental figure 2 Characterisation of PARP T-DNA mutant alleles.}

(a) Gene structures and confirmed insertion sites of PARP1, withparp1 -2 (SAIL_514F10) allele, PARP2 and parp2 -1 (GK380_E06) allele and PARP3 with the parp3 -1 (SALK108_092) allele. See text for precise positions. Positions of relevant primers pairs are indicated. Gene F/R (black arrows) = primers positioned to flank insertion site, qPCR (green arrows) = primers positioned downstream of insertion site. (b) PCR products from gDNA of triple mutant and Col-0 background plants confirm a homozygous tripleparp11x2-1x3-1 mutant. Gene-specific forward and reverse primers flanking the predicted insertion site amplify products from Col-0 gDNA but not gDNA from homozygous T-DNA insertion mutant gDNA. T-DNA insertion mutants amplify a product using $\mathrm{LB}$ primer and a gene specific primer. $\mathrm{F}=$ Forward primer, $\mathrm{R}=$ reverse primer, $\mathrm{LB}=\mathrm{T}-\mathrm{DNA}$ left border primer. (c) Relative abundance of PARP1, PARP2 andPARP3 transcripts in Col-0 and parp1-2x2-1x3-1 measured by qRT-PCR. N = 3 .

\section{Supplemental figure 3 Circadian rhythms of delayed fluorescence in parp and srt mutants}

Measurements of delayed chlorophyll fluorescence in otherwise constant light $\left(40-50 \mu\right.$ moles $\left.\mathrm{m}^{-2} \mathrm{~s}^{-1}\right)$ in Col- 0 background and (a) Col-0 $n=10$, parp1-2 $n=6$, (b) Col-0 $n=6$, parp2-1 $n=6$, (c) Col-0 $n=10$, parp3-1 $n=8$, (c) Col-0 $n=6$,parp1-2 $x$ 2-1 $x$ 3-1 $n=6$, (d) Col-0 $n=12$, srt1-4 hete $n=12$, and (e) Col-0 $n=12$, srt2-1 $n=9$. Delayed fluorescence was measured for $60 \mathrm{~s}$ post illumination. Cps $=$ counts per second. Error bars represent standard deviation.

Supplemental figure $4 S R T 1$ and $S R T 2$ are expressed in the aerial tissues of Arabidopsis. 
SRT1 and SRT2 transcript abundance in Arabidopsis organs measured using qRT-PCR and normalised to UBQ10 in RNA extracted from rosette leaves, cauline leaves, siliques, flowers and stem). $\mathrm{N}=3$.

Supplemental figure 5 parg1-1 is a long period circadian mutant

(a) $\operatorname{parg1-1}$ and (b) tej-1 seedlings along with appropriate backgrounds transformed with $C C A 1:: L U C$ and $C A B 2:: L U C$ respectively were sown in clusters of $3-5$ seeds on to $0.5 \mathrm{MS}$ in PVC tubing wells. Imaging of luciferase luminescence was performed in otherwise constant light. Two days before imaging clusters were dosed with $50 \mu \mathrm{l}$ of $2 \mathrm{mM}$ luciferin. Error bars represent standard deviation.

Supplemental figure 6 qRT-PCR measurement of selective transcripts in parp mutants.

Independent validation of RNAseq results. (a) RNAseq counts for selected transcripts found to have no change (PRR7), a decrease (GP2, SUS4), a moderate increase (CCA1) or a large increase (AHA8) in abundance. (b) qRT-PCR was used to measure the changes in abundance of the selected transcripts in parpmutants.. Plants were grown, harvested and all qRT-OCR analysis was performed independently of the RNAseq. $\mathrm{n}=3$.

Supplemental figure 7 Abundance of selected transcripts measured by qRT-PCR in sirtuin mutants compared to RNAseq data.

Genes encoding transcripts that were mis-regulated in srt1-4 heteRNA-seq were selected for further analysis by independent qRT-PCR in an attempt to confirm the major findings. At2G36970 was selected because it has a large decrease in abundance in RNAseq in the mutant.AT5G55580 and AT5G55540 were selected to represent transcripts that had smaller changes in abundance. AT5G62720, AT4G11830 and AT2G15880 were selected to represent large increases in abundance changes in srt1-4 hete the RNAseq data. (a, c) qRT-PCR of Col-0, srt1-4 hete, srt2-1 and microRNA lines SRT1amiR\#5 and SRT1amiR\#10 (b,d) RNAsequencing data from Col-0, srt1-4 hete and, srt2-1 as number of reads. Samples were obtained in the day $(\mathrm{a}, \mathrm{b}, \mathrm{ZT} 6)$ and night $(\mathrm{c}, \mathrm{d})$ ZT18. The qRT-PCR and RNAseq studies were performed independently on material grown and harvested separately.

Supplemental figure 8 srt1-4 hete and SRT1 artificial micro RNA lines have similar effects on transcript abundance.

(a) SRT1 abundance was measured by qRT-PCR in Col-0,srt1-1, srt1-2, srt1-3, srt2-1 T-DNA homozygous mutants and in srt1-4 heterozygous T-DNA insertion lines, andSRT1 artificial micro RNA line \#5 and \#10.

(b) Having confirmed knockdown of SRT1 in srt1-4 heterozygous T-DNA insertion lines and SRT1 artificial micro RNA line \#5 and \#10, transcript abundance of the products of four genes was measured by qRT-PCR. $\mathrm{n}=3$ (b). These four genes were identified as being mis-regulated insrt1-4 heterozygous lines (AT2G37678, AT5G02200 andAT2G42870) and both srt1-4 heterozygous lines andsrt2-1 (AT1G70290) when analysed by RNA-seq (Supplemental Table 5). Material for the qRT-PCR and RNAseq was grown and harvested separately and the experiments were independent. All plants were harvested during the day.

Supplemental Tables

Supplemental Table 1. Summary of all lines used

Mutants of Arabidopsis genes used in this study

Supplemental Table 2. Lists of primers for genotyping and qRT-PCR

List of primer sequences used for genotyping and list of primers for qRT-PCR analysis in this study.

F: Forward primer. R: reverse primer

Supplemental Table 3 European Bioinformatics Institute datasets used for comparison with results from RNAseq. 
Supplemental Table 4 List of differentially expressed genes inparp1-2, parp2-1, parp3-1, parp12x2-1x3-1 and Col-0 in day and night.

Supplemental Table 5 List of differentially expressed DF genes in srt1-4 hete, srt2-1, srt1-4 hete $x$ 2-1 and Col-0 in Day and Night.

Supplemental Table 6 List of genes and their descriptions in the four intersections in the Venn diagrams in Figure $9 \mathrm{~b}$.

Supplemental Table 7 Gene Ontology (GO) term enrichment in the gene lists from the four intersections in the Venn diagrams in Figure $9 \mathrm{~b}$.

Parsed Citations

Abdul-Awal S.M., Hotta C.T., Davey M.P., Dodd A.N., Smith A.G., Webb A.A.R. (2016) NO-mediated $\left[\mathrm{Ca}^{+}\right]$cyt increases depend on ADP-ribosyl cyclase activity in Arabidopsis. Plant Physiology 171: 623-631

Adams-Phillips L, Wan J, Tan X, Dunning F.M., Meyers B.C., Michelmore R.W., Bent A.F. (2008) Discovery of ADP-ribosylation and other plant defense pathway elements through expression profiling of four differentArabidopsis -Pseudomonas R-avr interactions. Molecular Plant-Microbe Interactions 21: 646-657

Adams-Phillips L, Briggs A.G., Bent A.F. (2010) Disruption of poly(ADP-ribosyl)ation mechanisms alters responses of Arabidopsis to biotic stress. Plant Physiology 152: 267-280

Ashburner M, Ball C.A., Blake J.A., Botstein D, Butler H, Cherry J.M., Davis A.P., Dolinski K, Dwight S.S., Eppig J.T., Harris M.A., Hill D.P., Issel-Tarver L, Kasarskis A, Lewis S, Matese J.C., Richardson J.E., Ringwald M, Rubin G.M., Sherlock G. (2000) Gene Ontology: tool for the unification of biology. Nature Genetics 25: 1546-1718.

Asher G, Gatfield D, Stratmann M, Reinke H, Dibner C, Kreppel F, Mostoslavsky R, Alt F.W., Schibler U. (2008) SIRT1 regulates circadian clock gene expression through PER2 deacetylation. Cell 134: 317-328

Asher G, Reinke H, Altmeyer M, Gutierrez-Arcelus M, Hottiger M.O., Schibler U. (2010) Poly(ADP-ribose) polymerase 1 participates in the phase entrainment of circadian clocks to feeding. Cell 142: 943-953

Ausin I, Alonso-Blanco C, Jarillo J.A., Ruiz-Garcia L, \& Martinez-Zapater J.M. (2004) Regulation of flowering time by FVE, a retinoblastoma-associated protein. Nature Genetics 36: 162-166

Bond D.M., Dennis E.S., Pogson B.J., \& Finnegan E.J. (2009) Histone acetylation, VERNALIZATION INSENSITIVE 3, FLOWERING LOCUS C, and the vernalization response. Molecular Plant 2: 724-737

Bravo-Sagua R, Parra V, López-Crisosto C, Diaz P, Quest A.F.G., \& Lavandero S. (2017). Calcium Transport and Signaling in Mitochondria. In Comprehensive Physiology, R. Terjung (Ed.). doi:10.1002/cphy.c160013

Carlson, M. (2020a). GO.db: A set of annotation maps describing the entire Gene Ontology. R package version 3.11.4.

Carlson, M. (2020b). org.At.tair.db: Genome wide annotation for Arabidopsis. R package version 3.11.4.

Chang H.C., \& Guarente L. (2013). SIRT1 mediates central circadian control in the SCN by a mechanism that decays with aging. Cell 153: 1448-1460

Chen Y.M., Shall S., \& Ofarrell M. (1994). Poly(ADP-ribose) polymerase in plant nuclei. European Journal of Biochemistry 224: 135-142

Das D.K., Mukherjee S, \& Ray D. (2010). Resveratrol and red wine, healthy heart and longevity. Heart Failure Reviews 15: 467-477

De Block M, Verduyn C, De Brouwer D, \& Cornelissen M. (2005). Poly(ADP-ribose) polymerase in plants affects energy homeostasis, cell death and stress tolerance. Plant Journal 41: 95-106 
Dodd A.N., Salathia N, Hall A, Kevei E, Toth R, Nagy F, Hibberd J.M., Millar A.J., \& Webb A.A.R. (2005). Plant circadian clocks increase photosynthesis, growth, survival, and competitive advantage. Science 309: 630-633

Dodd A.N., Gardner M.J., Hotta C.T., Hubbard K.E., Dalchau N, Love J, Assie J.M., Robertson F.C., Jakobsen M.K., Goncalves J, Sanders D, \& Webb A.A.R. (2007). The Arabidopsis circadian clock incorporates a cADPR-based feedback loop. Science 318, 1789-1792.

Falcon S, \& Gentleman R. (2007). Using GOstats to test gene lists for GO term association. Bioinformatics 23:257-8, 2007.

Farinas B, \& Mas P. (2011). Functional implication of the MYB transcription factor RVE8/LCL5 in the circadian control of histone acetylation. Plant Journal 66: 318-329

Feng B, Liu C, de Oliveira M.V.V., Intorne A.C., Li B, Babilonia K, Filho G, Shan L \& Ping He. (2015). Protein Poly(ADP-ribosyl)ation Regulates Arabidopsis Immune Gene Expression and Defense Responses. PLoS Genet 11(1): e1004936. doi:10.1371

Graf A, Schlereth A, Stitt M, \& Smith A.M. (2010). Circadian control of carbohydrate availability for growth in Arabidopsis plants at night. Proceedings of the National Academy of Sciences of the United States of America 107: 9458-9463

Gutierez R.A., Stokes T.L., Thum K, Xu X, Obertello M, Katari M.S., Tanurdzic M, Dean A, Nero D.C., McClung C.R., \& Coruzzi G.M. (2008). Systems approach identifies an organic nitrogen-responsive gene network that is regulated by the master clock control gene CCA1. Proceedings of the National Academy of Sciences of the United States of America 105: 4939-4944

Hardcastle T.J., \& Kelly K. (2010). baySeq: Empirical Bayesian methods for identifying differential expression in sequence count data. BMC Bioinformatics 11:422

He Y.H., Michaels S.D., \& Amasino R.M. (2003). Regulation of flowering time by histone acetylation in Arabidopsis. Science 302: 1751-1754

Hearn T.J., Abdul-Awal S.M., Stanton C.R., Moeller R.M., Marti C.M.C., Haydon, M.J., Theodoulou, F.L., Hannah, M.A. \& Webb A.A.R. (2018). BIG regulates dynamic adjustment of circadian period in Arabidopsis thaliana. Plant Physiology 178, 358-371.

Heller B, Wang Z.Q., Wagner E.F., Radons J, Burkle A, Fehsel K, Burkart V, \& Kolb H (1995). Inactivation of the poly(ADP-ribose) polymerase gene affects oxygen radical and nitric-oxide toxicity in islet cells. Journal of Biological Chemistry 270: 11176-11180

Hunt L, Lerner F, \& Ziegler M. (2004). NAD - new roles in signalling and gene regulation in plants. New Phytologist 163: 31-44

Imai S, Armstrong C.M., Kaeberlein M, \& Guarente L. (2000). Transcriptional silencing and longevity protein Sir2 is an NAD-dependent histone deacetylase. Nature 403: 795-800

Jaspers P, Overmyer K, Wrzaczek M, Vainonen J.P., Blomster T, Salojarvi J, Reddy RA, \& Kangasjarvi J. (2010). The RST and PARP-like domain containing SRO protein family: analysis of protein structure, function and conservation in land plants. BMC Genomics 11

Kim H.J., Hyun Y, Park J.Y., Park M.J., Park M.K., Kim M.D., Lee M.H., Moon J, Lee I, \& Kim J. (2004). A genetic link between cold responses and flowering time through FVE in Arabidopsis thaliana. Nature Genetics 36: 167-171

Kerwin R.E., Jimenez-Gomez J.M., Fulop D, Harmer S.L., Maloof J.N., \& Kliebenstein D.J. (2011). Network quantitative trait loci mapping of circadian clock outputs identifies metabolic pathway-to-clock linkages in Arabidopsis. Plant Cell 23: 471-485 
Krishnakumar R, \& Kraus W.L. (2010). The PARP side of the nucleus: molecular actions, physiological outcomes, and clinical targets. Molecular Cell 39: 8-24

Kumar V, \& Takahashi J.S. (2010). PARP around the clock. Cell 142: 841-843

Lagouge M, Argmann C, Gerhart-Hines Z, Meziane H, Lerin C, Daussin F, Messadeq N, Milne J, Lambert P, Elliott P, Geny B, Laakso M, Puigserver P, \& Auwerx J. (2006). Resveratrol improves mitochondrial function and protects against metabolic disease by activating SIRT1 and PGC-1 alpha. Cell 127: 1109-1122

Lai A.G., Doherty C.J., Mueller-Roeber B, Kay S.A., Schippers J.H.M., \& Dijkwel P.P. (2012). CIRCADIAN CLOCK-ASSOCIATED 1 regulates ROS homeostasis and oxidative stress responses. Proceedings of the National Academy of Sciences of the United States of America 109: 17129-17134

Landry J, Sutton A, Tafrov S.T., Heller R.C., Stebbins J, Pillus L, \& Sternglanz R. (2000). The silencing protein SIR2 and its homologs are NAD-dependent protein deacetylases. Proceedings of the National Academy of Sciences of the United States of America 97: 5807-5811

Langmead, B., \& Salzberg, S. L. (2012). Fast gapped-read alignment with Bowtie 2. Nature Methods, 9, $357-359$.

Liu XY, Wei W, Zhu W.J., Su L.F., Xiong Z.Y., Zhou M, Zheng Y, \& Zhou D.X. (2017). Histone deacetylase AtSRT1 links metabolic flux and stress response in Arabidopsis..Molecular Plant 10 (12): 1510

Lu Y, Gehan J.P., \& Sharkey T.D. (2005). Daylength and circadian effects on starch degradation and maltose metabolism. Plant Physiology 138: 2280-2291

Marti Ruiz M.C., Hubbard K.E., Gardner M.J., Aubry S, Hotta C.T., Mohd-Noh, NI, Lan H, Robertson F.C., Hearn T.J., Jung H.J., Tsai Y-C, Dodd A.N., Hannah M, Carre IA, Braam J, \& Webb A.A.R. (2018). Circadian oscillations of cytosolic free calcium regulate the Arabidopsis circadian clock. Nature Plants 4, 690-698

Malapeira J, Crhak Khaitova L, \& Mas P. (2012). Ordered changes in histone modifications at the core of the Arabidopsis circadian clock. Proceedings of the National Academy of Sciences of the United States of America 109: 21540-21545

Mombaerts, L., Carignano, A., Robertson F.C., Hearn, T.J., Junyang, J., Hayden D., Rutterford, Z., Hotta, C.T., Hubbard, K.E., Yuan Y., Hannah M.A., Goncalves, J. \& Webb A.A.R. (2019). Differentially Expressed Systems (DES) identifies Dynamical Differential Expression (DyDE) Reveals the Period Control Mechanisms of the Arabidopsis Circadian Oscillator. PLOS Comput Biol 15: e1006674

Nakahata Y, Kaluzova M, Grimaldi B, Sahar S, Hirayama J, Chen D, Guarente L.P., \& Sassone-Corsi P. (2008). The $\mathrm{NAD}^{+}$-dependent deacetylase SIRT1 modulates CLOCK-mediated chromatin remodeling and circadian control. Cell 134: 329-340

Nakahata Y, Sahar S, Astarita G, Kaluzova M, \& Sassone-Corsi P. (2009). Circadian control of the NAD ${ }^{+}$ salvage pathway by CLOCK-SIRT1. Science 324: 654-657

Oike H, \& Kobori M. (2008). Resveratrol regulates circadian clock genes in Rat-1 fibroblast cells. Bioscience Biotechnology and Biochemistry 72: 3038-3040

Ossowski S, Schwab R, \& Weigel D. (2008). Gene silencing in plants using artificial microRNAs and other small RNAs. Plant Journal. 53: 674-690.

Panda S, Poirier G.G., \& Kay S.A. (2002). tej defines a role for poly(ADP-ribosyl)ation in establishing period length of the Arabidopsis circadian oscillator. Developmental Cell 3: 51-61

Perales M, \& Mas P. (2007). A functional link between rhythmic changes in chromatin structure and the Arabidopsis biological clock. Plant Cell 19: 2111-2123 
Perraud A.L., Takanishi C.L., Shen B, Kang S, Smith M.K., Schmitz C, Knowles H.M., Ferraris D, Li W.X., Zhang J, Stoddard B.L., \& Scharenberg A.M. (2005). Accumulation of free ADP-ribose from mitochondria mediates oxidative stress-induced gating of TRPM2 cation channels. Journal of Biological Chemistry 280: 6138-6148

Pifferi F, Dal-Pan A, Menaker M, \& Aujard F. (2011). Resveratrol dietary supplementation shortens the free-running circadian period and decreases body temperature in a prosimian primate. Journal of Biological Rhythms 26: 271-275

Roberts A, \& Pachter L. (2013). Streaming fragment assignment for real-time analysis of sequencing experiments. Nat Methods 10: 71-73

Rissel D, Heym P.P., Thor K, Brandt W, Wessjohann L.A., \& Peiter E. (2017). No silver bullet - canonical poly(ADP-ribose) polymerases (PARPs) are no universal factors of abiotic and biotic stress resistance of Arabidopsis thaliana. Frontiers in Plant Science 8:59

Schreiber V, Dantzer F, Ame J.C., \& de Murcia G. (2006). Poly(ADP-ribose): novel functions for an old molecule. Nature Reviews Molecular Cell Biology 7: 517-528

Shen, L, (2020). GeneOverlap: Test and visualize gene overlaps. R package version 1.24.0. http://shenlabsinai.github.io/shenlab-sinai/

Smith J.S., Brachmann C.B., Celic I, Kenna M.A., Muhammad S, Starai V.J., Avalos J.L., EscalanteSemerena J.C., Grubmeyer C, Wolberger C, \& Boeke J.D. (2000). A phylogenetically conserved NAD ${ }^{+}$ dependent protein deacetylase activity in the Sir2 protein family. Proceedings of the National Academy of Sciences of the United States of America 97: 6658-6663

Song H.R., \& Noh Y.S. (2012). Rhythmic oscillation of histone acetylation and methylation at the Arabidopsis central clock loci. Molecules and Cells 34: 279-287

Vanderauwera S, De Block M, van de Steene N, de Cottet B.V., Metzlaff M, \& Van Breusegem F. (2007). Silencing of poly(ADP-ribose) polymerase in plants alters abiotic stress signal transduction. Proceedings of the National Academy of Sciences of the United States of America 104: 15150-15155

Wang C, Gao F, Wu J, Dai J, Wei C, \& Li Y. (2010). Arabidopsis Putative Deacetylase AtSRT2 regulates basal defense by suppressing PAD4, EDS5 and SID2 expression. Plant and Cell Physiology 51: 1291-1299

Wang L, Kim J, \& Somers D.E. (2013). Transcriptional corepressor TOPLESS complexes with pseudoresponse regulator proteins and histone deacetylases to regulate circadian transcription. Proceedings of the National Academy of Sciences of the United States of America 110: 761-766.

Webb A.A.R., Seki M, Satake A. \& Caldana C. (2019). Continuous dynamic adjustment of the plant circadian oscillator. Invited Perspective for Nature Communications 10, 550.

Xiao J, Zhang H, Xing L, Xu S, Liu H, Chong K, \& Xu Y. (2013). Requirement of histone acetyltransferases HAM1 and HAM2 for epigenetic modification of FLC in regulating flowering in Arabidopsis. Journal of Plant Physiology 170: 444-451

Zhang F, Wang L, Ko E.E., Shao K, \& Qiao H. (2018). Histone deacetylases SRT1 and SRT2 interact with ENAP1 to mediate ethylene-induced transcriptional repression. The Plant Cell 30: 153-166

Zhang N, Meng Y, Li X, Zhou Y, Ma L, Fu L, Schwarzlander L, Liu H, \& Xiong Y. (2019). Metabolitemediated TOR signaling regulates the circadian clock in Arabidopsis. Proceedings of the National Academy of Sciences, 201913095. https://doi.org/10.1073/pnas.1913095116 

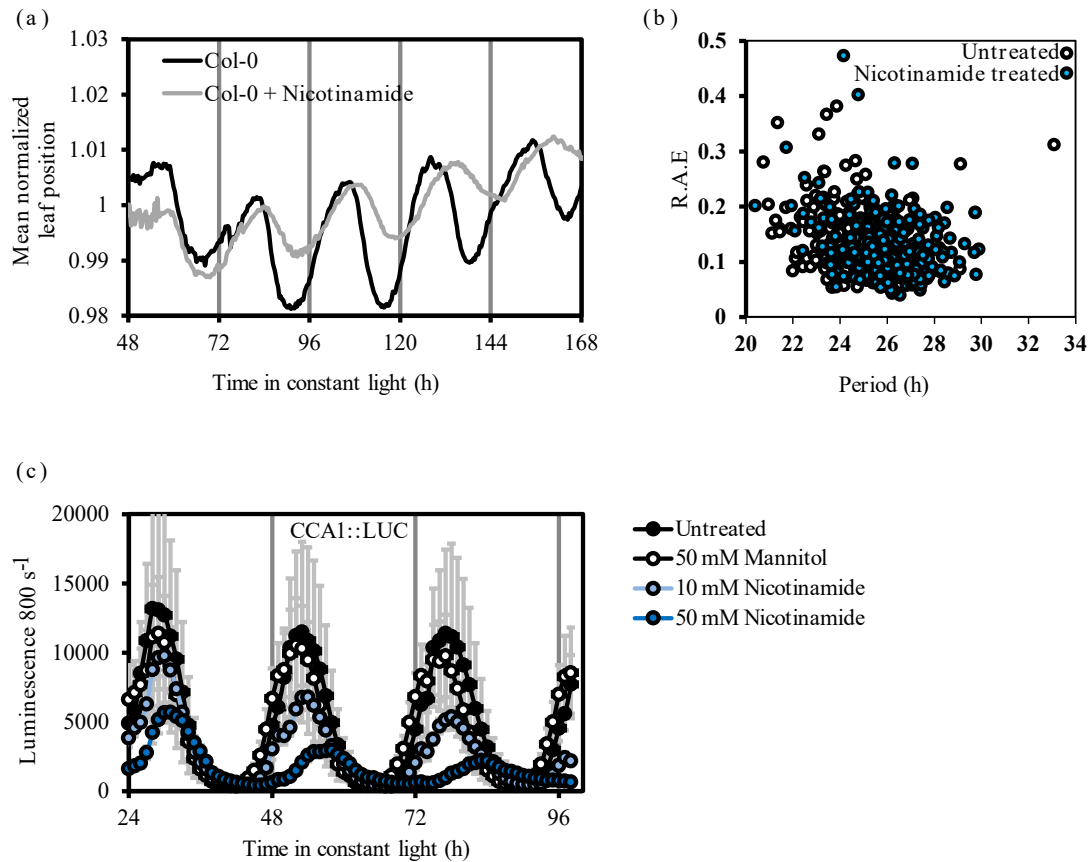

$\underset{-0 \text { Untreated }}{\longrightarrow} 50 \mathrm{mM}$ Mannitol - $10 \mathrm{mM}$ Nicotinamide - $-50 \mathrm{mM}$ Nicotinamide 
Kim et al Figure 2

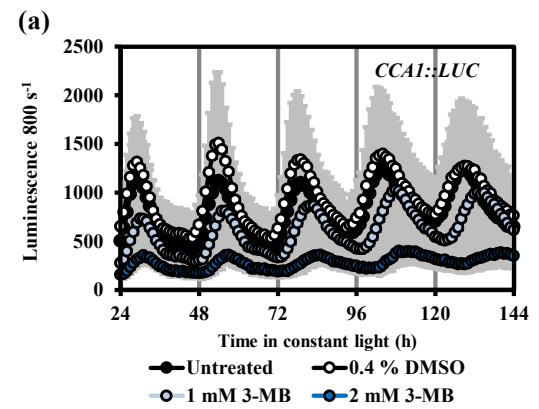

(b)

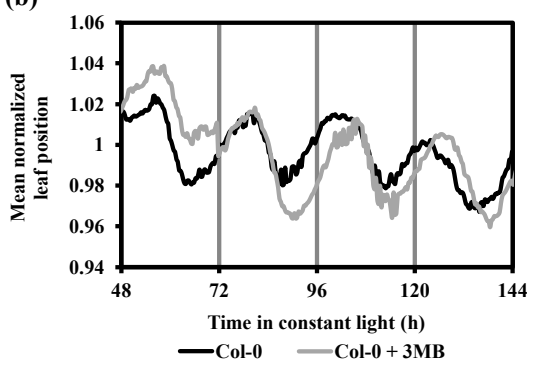


Kim et al Figure 3

(a)
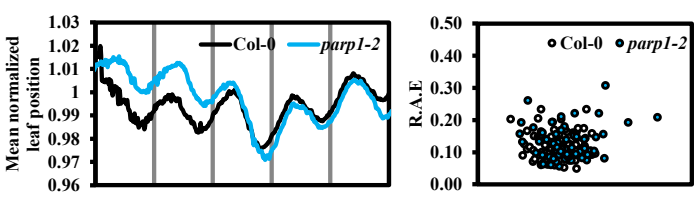

(b)

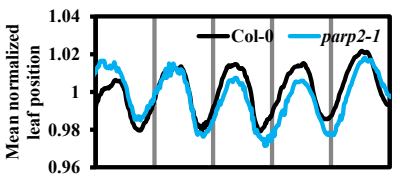

(c)

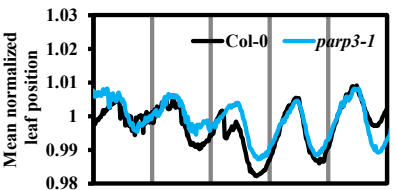

(d)
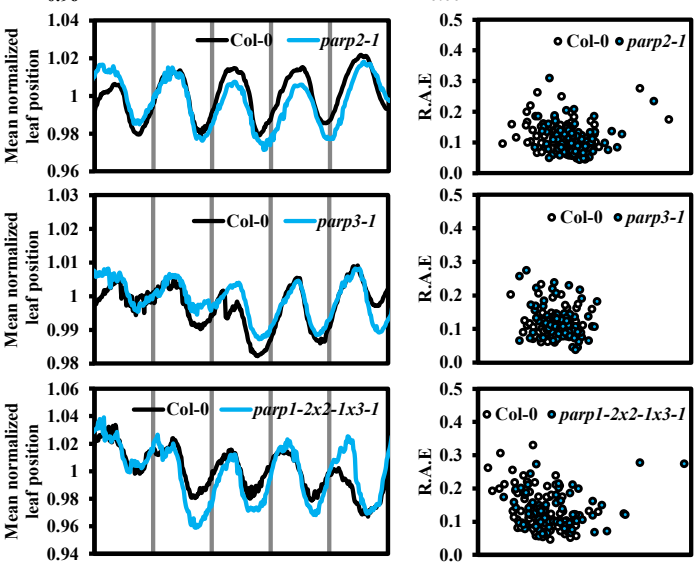

(e)
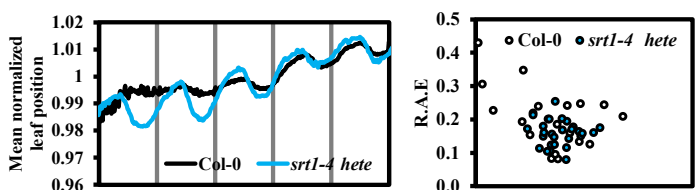

(f)
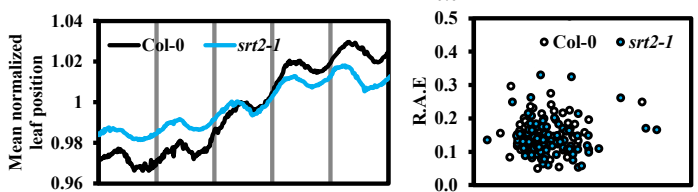

(g)

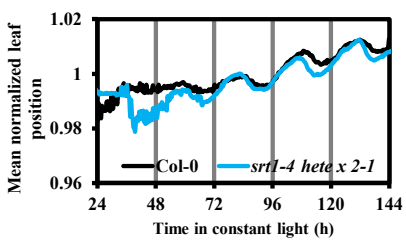

0.5 ० Col-0 osrtl-4 hete $x$ 2-1

0.410 .0

$0.3 .0 \%$

0.2 \% \%

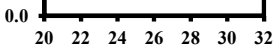

Period (h) 
Kim et al Figure 4

A

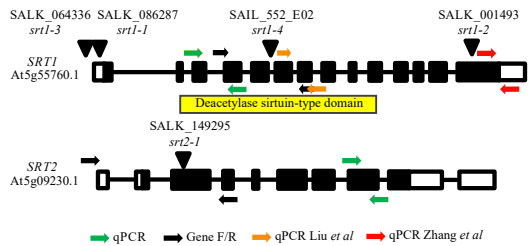

B

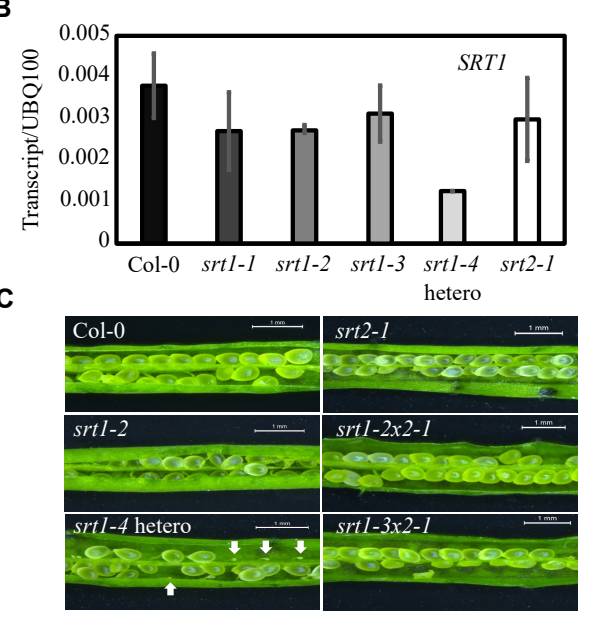

D

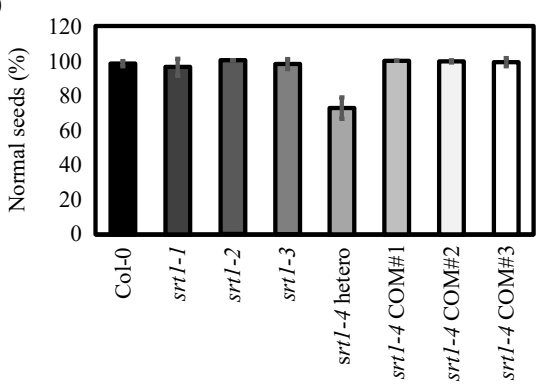


Kim et al Figure 5

(a)

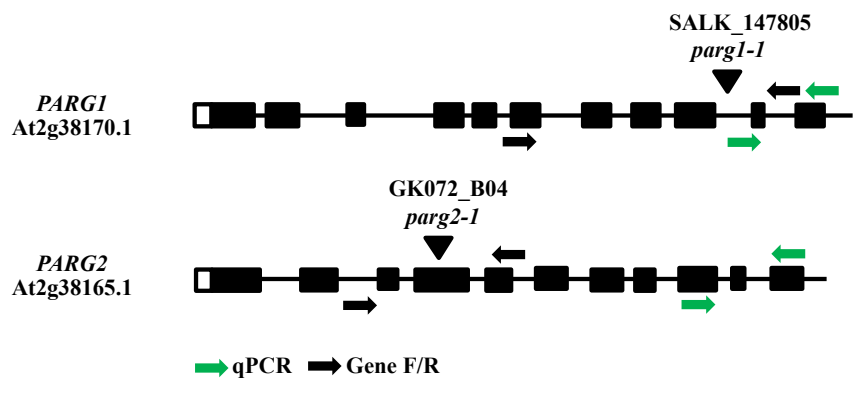

(b)

(c)
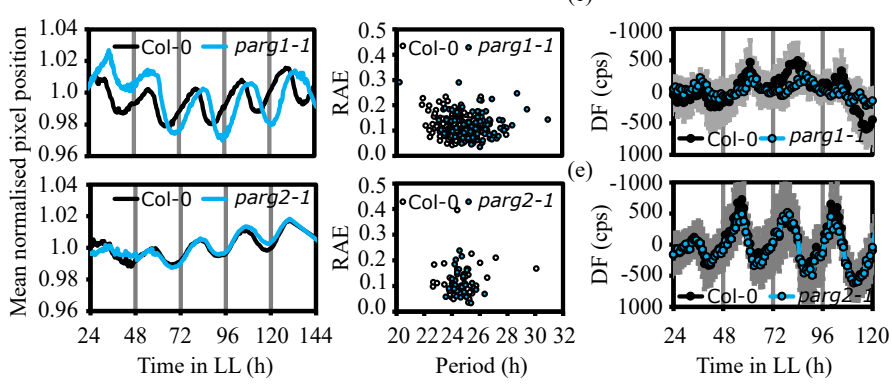
Kim et al Figure 6
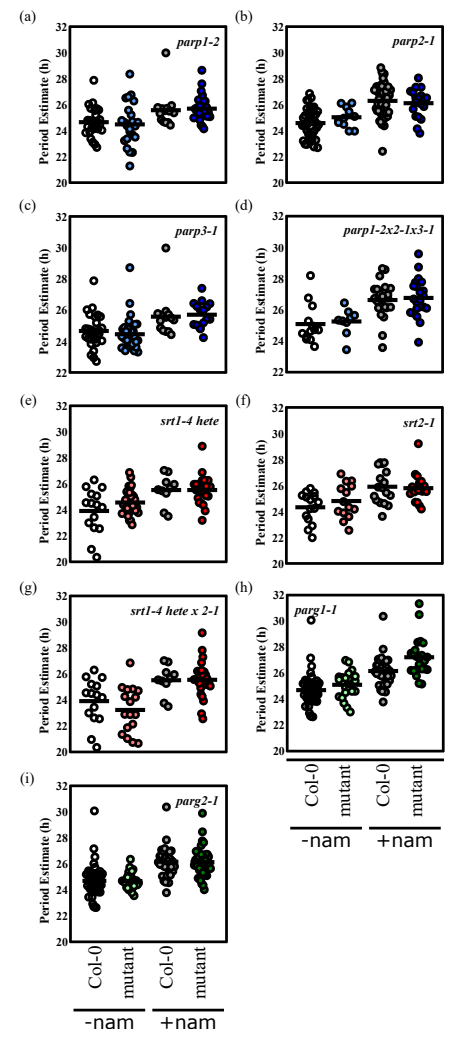
Kim et al Figure 7
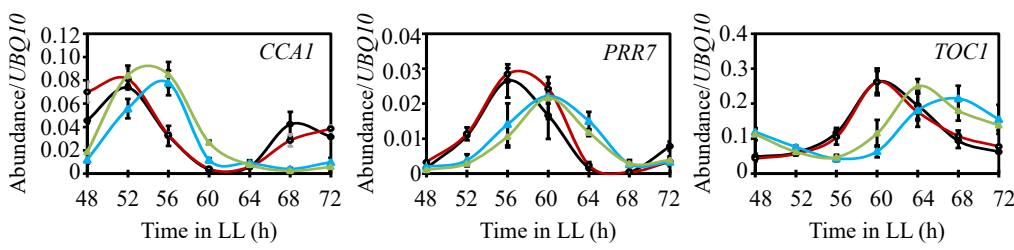

$\rightarrow$ Col-0 $\rightarrow$ Col-0 + nicotinamide

$\rightarrow$ parp1-2x2-1x3-1 $\rightarrow$ parp1-2x2-1x3-1 + nicotinamide 
Kim et al., Figure 8

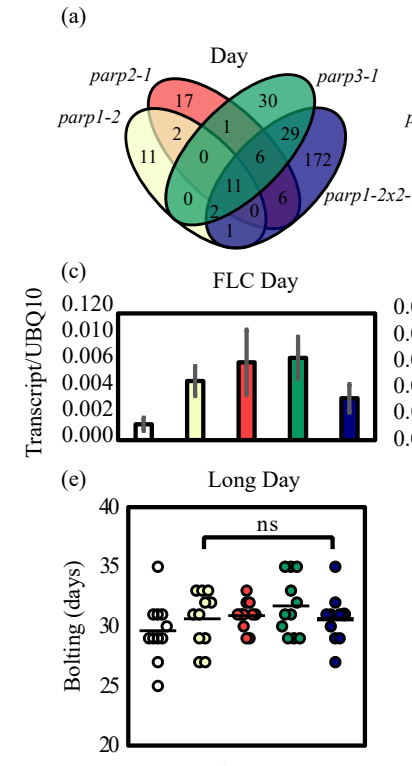

(b)

parp2-1 Night
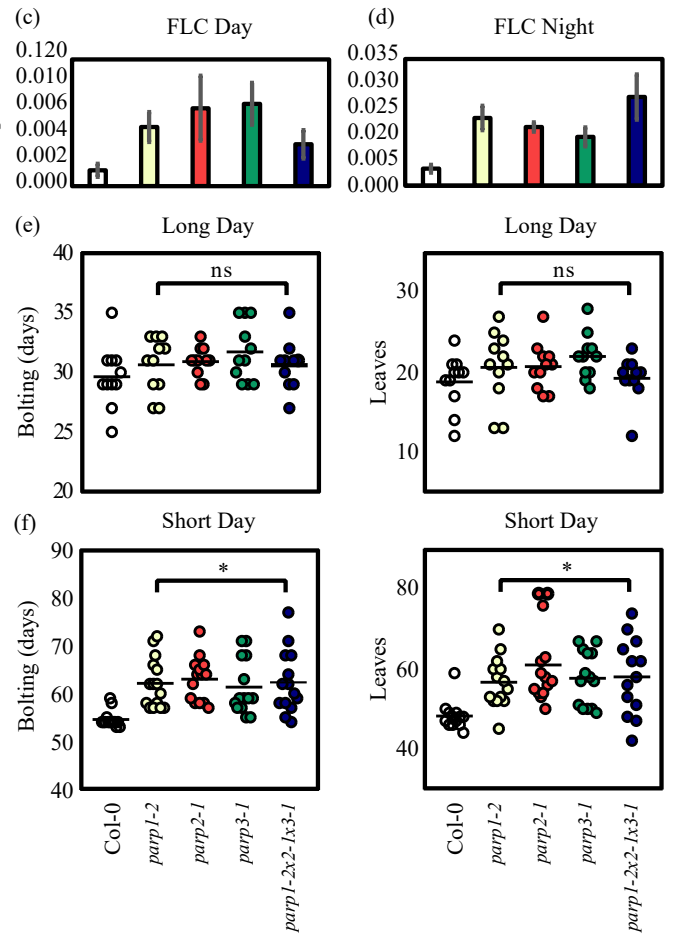

Long Day

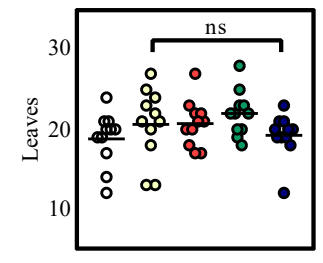

Short Day

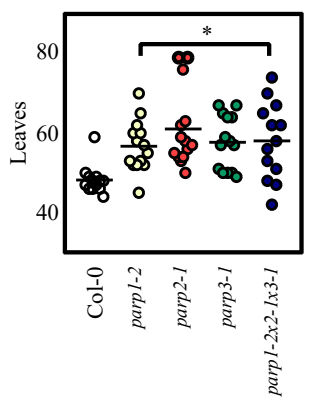


Kim et al Figure 9

(a)
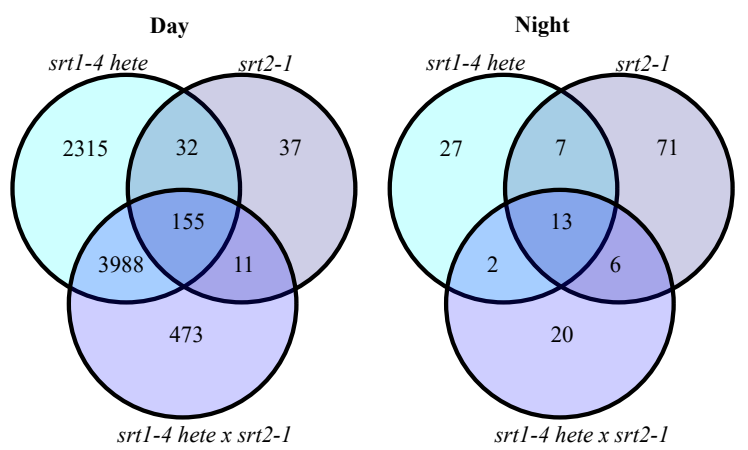

(b)

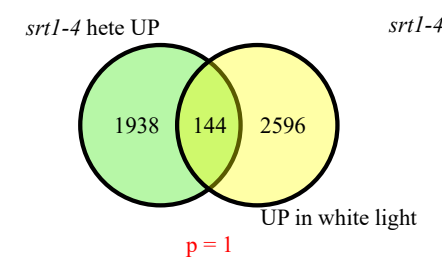

srt1-4 hete DOWN

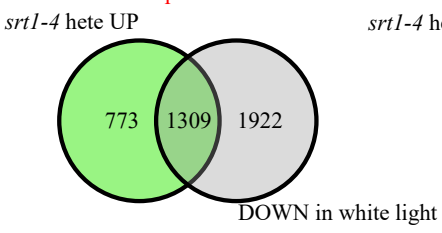

$\mathrm{p}=1.68 \mathrm{e}-232$

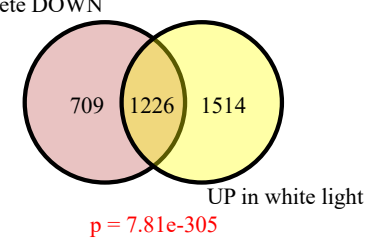

(c)
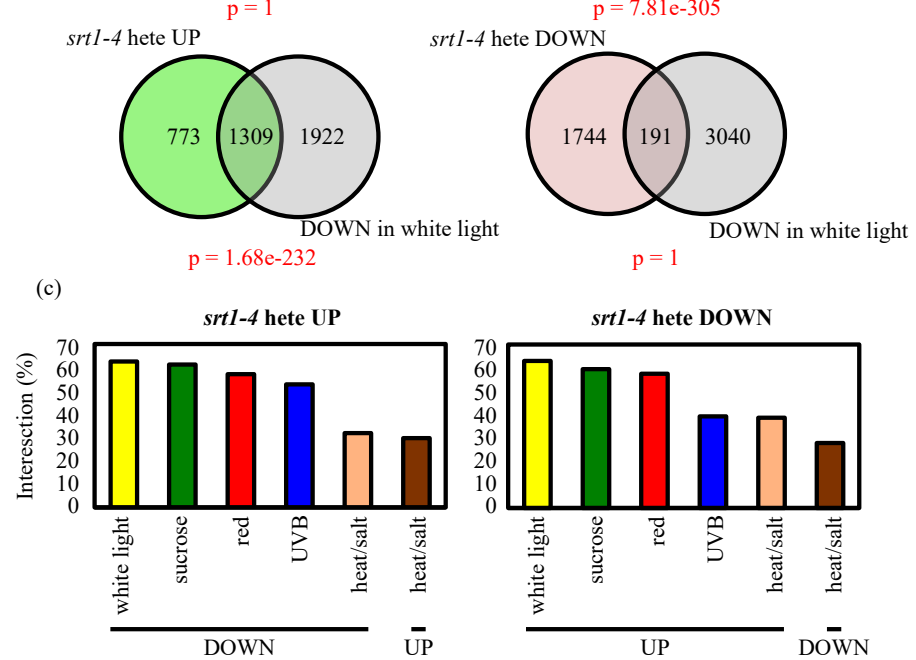